\title{
STOCHASTIC “BEADS ON A STRING” IN THE ACCRETION TAIL OF ARP 285
}

\author{
Beverly J. Smith ${ }^{1}$, Curtis Struck ${ }^{2}$, Mark Hancock ${ }^{1}$, Mark L. Giroux ${ }^{1}$, Philip N. Appleton ${ }^{3}$, Vassilis Charmandaris ${ }^{4,6}$, \\ William Reach ${ }^{5}$, Sabrina Hurlock ${ }^{1}$, and JeOng-Sun Hwang ${ }^{2}$ \\ ${ }^{1}$ Department of Physics, Astronomy, and Geology, East Tennessee State University, Johnson City, TN 37614, USA; smithbj@etsu.edu, hancockm@etsu.edu, \\ girouxm@etsu.edu, and zshh7@imail.etsu.edu \\ ${ }^{2}$ Department of Physics and Astronomy, Iowa State University, Ames, IA 50011, USA; curt@iastate.edu, jshwang@iastate.edu \\ ${ }^{3}$ NASA Herschel Science Center, California Institute of Technology, Pasadena, CA 91125, USA; apple@ipac.caltech.edu \\ ${ }^{4}$ Department of Physics, University of Crete, Heraklion Greece 71003, Greece; vassilis@ physics.uoc.gr \\ ${ }^{5}$ Spitzer Science Center, California Institute of Technology, Pasadena, CA 91125, USA; reach@ipac.caltech.edu \\ Received 2007 September 5; accepted 2008 February 24; published 2008 May 15
}

\begin{abstract}
We present Spitzer infrared, Galaxy Evolution Explorer UV, and Sloan Digitized Sky Survey and Southeastern Association for Research in Astronomy optical images of the peculiar interacting galaxy pair Arp 285 (NGC 2856/4), and compare with a new numerical model of the interaction. We estimate the ages of clumps of star formation in these galaxies using population synthesis models, carefully considering the uncertainties on these ages. This system contains a striking example of "beads on a string": a series of star-formation complexes $\sim 1 \mathrm{kpc}$ apart. These "beads" are found in a tail-like feature that is perpendicular to the disk of NGC 2856, which implies that it was formed from material accreted from the companion NGC 2854. The extreme blueness of the optical/UV colors and redness of the mid-infrared colors implies very young stellar ages ( 4-20 Myr) for these star-forming regions. Spectral decomposition of these "beads" shows excess emission above the modeled stellar continuum in the $3.6 \mu \mathrm{m}$ and $4.5 \mu \mathrm{m}$ bands, indicating either contributions from interstellar matter to these fluxes or a second older stellar population. These clumps have $-12.0<M_{\mathrm{B}}<-10.6$, thus they are less luminous than most dwarf galaxies. Our model suggests that bridge material falling into the potential of the companion overshoots the companion. The gas then piles up at apogalacticon before falling back onto the companion, and star formation occurs in the pile-up. There was a time delay of $\sim 500$ Myr between the point of closest approach between the two galaxies and the initiation of star formation in this feature. A luminous $\left(M_{\mathrm{B}} \sim-13.6\right)$ extended $(\mathrm{FWHM} \sim 1.3 \mathrm{kpc})$ "bright spot" is visible at the northwestern edge of the NGC 2856 disk, with an intermediate stellar population (400-1500 Myr). Our model suggests that this feature is part of a expanding ripple-like "arc" created by an off-center ring-galaxy-like collision between the two disks.
\end{abstract}

Key words: galaxies: individual (Arp 285) - galaxies: interactions - galaxies: starburst

\section{INTRODUCTION}

Galaxy evolution is strongly driven by interactions and mergers between galaxies. Interactions can produce tidal tails and bridges (Toomre \& Toomre 1972), increase star-formation rates (Kennicutt et al. 1987; Bushouse et al. 1988), and trigger the formation of young super-star clusters (Holtzman et al. 1992, 1996). Tidal material can contribute to the intergalactic medium (Morris \& van den Bergh 1994) and to intergalactic starlight (Feldmeier et al. 2002). Gas-rich galaxy mergers can produce ultra-luminous infrared galaxies (Soifer et al. 1987; Smith et al. 1987; Sanders et al. 1988), while concentrations of stars and gas in tidal features may become independent dwarf galaxies (Barnes \& Hernquist 1992; Elmegreen et al. 1993).

The key to understanding these processes is careful comparison of multi-wavelength observations of nearby galaxies with dynamical models. Since interactions and mergers are even more common at high redshift than in the local universe (e.g., Abraham \& van den Bergh 2001), detailed studies of nearby interacting systems are important for interpreting high redshift surveys. Such studies can provide information on the timescale of the interaction, the history of gas compression in different regions, star-formation triggering, dissipation in the gas, multiple bursts of star formation, and mass transfer between galaxies (Struck

6 IESL/Foundation for Research and Technology, Hellas, GR-71110, Heraklion, Greece and Chercheur Associé, Observatoire de Paris, F-75014, Paris, France.
\& Smith 2003; Struck et al. 2005; Smith et al. 2005a; Hancock et al. 2007). Computer simulations can provide predictions of the distribution of star formation, which can be compared to observational results to estimate the effects of compression strength, duration, and other factors (e.g., Struck \& Smith 2003).

To study star-formation enhancement in pre-merger interacting systems, we obtained mid-infrared observations with the Spitzer telescope (Werner et al. 2004) for three dozen interacting galaxies selected from the Arp (1966) Atlas of Peculiar Galaxies (the "Spirals, Bridges, and Tails" (SB\&T) sample; Smith et al. 2007). We have completed detailed multi-wavelength studies of three of the galaxy pairs in the SB\&T sample, and have constructed matching hydrodynamical models of their encounters: Arp 284 (Smith et al. 1997; Struck \& Smith 2003; Smith et al. 2005b), Arp 107 (Smith et al. 2005a), and Arp 82 (Hancock et al. 2007). A similar study of the interacting pair IC 2163/ NGC 2207 was presented by Struck et al. (2005) and Elmegreen et al. (2006), while Arp 24 was studied by Chen \& Wu (2007).

In the current paper, we describe a multi-wavelength study of another of the SB\&T systems, the interacting galaxy pair Arp 285 (NGC 2856/4), and compare with a new numerical model of the interaction. The more northern galaxy in this widely separated pair, NGC 2856, has a peculiar tail-like feature extending out perpendicular to the disk (Figure 1). Toomre \& Toomre (1972) suggested that this feature is material from the southern galaxy NGC 2854, which has accreted onto NGC 2856 via the bridge. The presence of a massive $\mathrm{H}$ I counterpart to this 
Table 1

Total Galaxian Magnitudes ${ }^{\mathrm{a}}$

\begin{tabular}{lcccccccccccc}
\hline \hline ID & $\begin{array}{c}\text { FUV } \\
(\mathrm{mag})\end{array}$ & $\begin{array}{c}\text { NUV } \\
(\mathrm{mag})\end{array}$ & $\begin{array}{c}u \\
(\mathrm{mag})\end{array}$ & $\begin{array}{c}g \\
(\mathrm{mag})\end{array}$ & $\begin{array}{c}r \\
(\mathrm{mag})\end{array}$ & $\begin{array}{c}i \\
(\mathrm{mag})\end{array}$ & $\begin{array}{c}z \\
(\mathrm{mag})\end{array}$ & $\begin{array}{c}F_{3.6 \mu \mathrm{m}} \\
(\mathrm{mJy})\end{array}$ & $\begin{array}{c}F_{4.5 \mu \mathrm{m}} \\
(\mathrm{mJy})\end{array}$ & $\begin{array}{c}F_{5.8 \mu \mathrm{m}} \\
(\mathrm{mJy})\end{array}$ & $\begin{array}{c}F_{8.0 \mu \mathrm{m}} \\
(\mathrm{mJy})\end{array}$ & $\begin{array}{c}F_{24 \mu \mathrm{m}} \\
(\mathrm{mJy})\end{array}$ \\
\hline NGC 2856 & 17.17 & 16.56 & 14.76 & 13.38 & 12.70 & 12.32 & 12.11 & 62.0 & 42.0 & 130.4 & 367.5 & 622.5 \\
NGC 2854 $^{16.49}$ & 15.95 & 14.76 & 13.49 & 12.88 & 12.53 & 12.33 & 41.4 & 26.2 & 73.9 & 189.9 & 184.4 \\
ANON $^{\mathrm{b}}$ & 20.91 & 20.78 & 19.96 & 18.99 & 18.99 & 18.71 & 18.70 & 0.12 & 0.08 & $<0.17$ & $<0.15$ & $<0.60$ \\
\hline
\end{tabular}

Notes.

a The statistical uncertainties in the optical and UV magnitudes are typically $\sim 0.01$ mag. The Spitzer uncertainties

are as given in Smith et al. (2007).

b At $9^{\mathrm{h}} 24^{\mathrm{m}} 2.9^{\mathrm{s}},+49^{\circ} 14^{\prime} 41^{\prime \prime}(\mathrm{J} 2000)$.

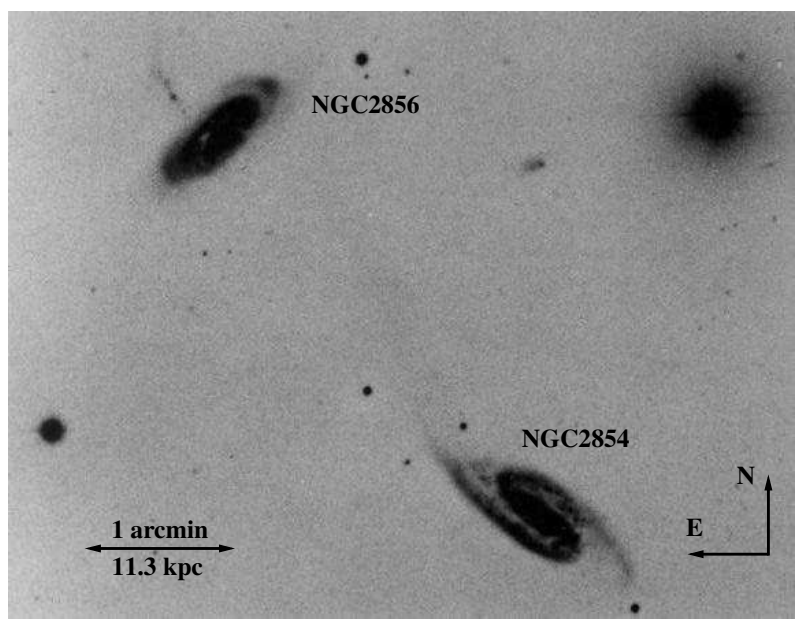

Figure 1. The Arp (1966) image of the wide galaxy pair Arp 285 (NGC 2856/4). The northern galaxy NGC 2856 has an unusual "tail-"like feature extending out perpendicular to the disk from the middle of the disk. Toomre \& Toomre (1972) suggested that this is the material from the bridge/companion, which is accreting onto NGC 2856. Note that this tail appears clumpy in this image. Note the "bright spot' in the northwestern edge of the NGC 2856 disk.

tail and the $\mathrm{H}$ I velocity field support this hypothesis (Chengalur et al. 1994, 1995). The Spitzer $3.6 \mu \mathrm{m}-8.0 \mu \mathrm{m}$ broadband infrared color of the NGC 2856 tail is the reddest of all the tidal features in the SB\&T sample (Smith et al. 2007), implying a very young stellar population.

In the current study, we investigate star formation in Arp 285 by combining our Spitzer mid-infrared images with ultraviolet images from the Galaxy Evolution Explorer (GALEX) mission (Martin et al. 2005) and optical images from the Sloan Digitized Sky Survey (SDSS) (Abazajian et al. 2003) and the Southeastern Association for Research in Astronomy (SARA) telescope. ${ }^{7}$ We also compare with the Two Micron All Sky Survey (2MASS) Atlas near-infrared images of Arp 285 (Cutri et al. 2006). Arp 285 is relatively nearby, at a distance of $39 \mathrm{Mpc}\left(H_{0}=\right.$ $75 \mathrm{~km} \mathrm{~s}^{-1} \mathrm{Mpc}^{-1}$ ).

\section{OBSERVATIONS}

The Spitzer infrared observations and data reductions are described in detail in Smith et al. (2007). The data used includes broadband $3.6 \mu \mathrm{m}, 4.5 \mu \mathrm{m}, 5.8 \mu \mathrm{m}$, and $8.0 \mu \mathrm{m}$ images from the Infrared Array Camera (IRAC; Fazio et al. 2004), with spatial resolutions of $1.15-2$.'0, a pixel size of $1^{\prime \prime} .2$, and a field of view of 7.8 $\times 12^{\prime} .6$. A $24 \mu \mathrm{m}$ image of

7 http://astro.fit.edu/sara/sara.html.
Arp 285 was also obtained with the Multiband Imaging Photometry for Spitzer (MIPS; Rieke et al. 2004), however, it has pronounced artifacts from the point-spread function (see image in Smith et al. 2007). Because of these artifacts, this image is only useful for determining total galaxian fluxes, not fluxes for individual clumps or tidal features. Thus it is not used in this analysis.

Arp 285 was observed as part of the SDSS in the ugriz optical filters (effective wavelengths of $3560 \AA, 4680 \AA, 6180 \AA$, $7500 \AA$, and $8870 \AA$, respectively). These images have a pixel size of 0.40 and a field of view of $13.5 \times 9.8$. The two galaxies in the pair are in two different SDSS fields of view. The FWHM point-spread function is $\sim 1^{\prime \prime}$. 2 , based on stars in the field.

Arp 285 was also observed with the SARA $0.9 \mathrm{~m}$ optical telescope on 2006 January 29, in partly cloudy weather. An $1152 \times 770$ Apogee Alta CCD with a pixel size of 0!'64 pixel $^{-1}$ was used, giving a field of view of $12.3 \times 8.2$. A total of three $600 \mathrm{~s}$ exposures were made in a broadband $R$ filter, along with seven $600 \mathrm{~s}$ images in a red-shifted $\mathrm{H} \alpha$ filter centered at $664 \mathrm{~nm}$ with a FWHM of $7 \mathrm{~nm}$. For Arp 285, this filter contains both $\mathrm{H} \alpha$ and the [N II] $\lambda \lambda 6548,6583$ line. The SARA data were reduced in the standard way using the Image Reduction and Analysis Facility (IRAF ${ }^{8}$ ) software. Continuum subtraction was accomplished using the scaled $R$-band image.

Arp 285 was observed in a near-ultraviolet (NUV) broadband filter (1750-2800 A) by GALEX as part of the GALEX Medium Imaging Survey (MIS) (Martin et al. 2005). The MIS image had a total integration time of $813 \mathrm{~s}$. Arp 285 was also observed in the far-ultraviolet (FUV) (1350-1705 $\AA$ ) as part of the GALEX All-Sky Survey (Martin et al. 2005), with a shorter exposure time of $112 \mathrm{~s}$. The GALEX spatial resolution is $\sim 5^{\prime \prime}$, with a pixel size of $1^{\prime \prime} .5$. The field of view is circular, with a $1.2^{\circ}$ diameter.

The total magnitudes for NGC 2854 and NGC 2856 in the various filters are given in Table 1.

\section{THE MORPHOLOGY OF ARP 285}

\section{1. $N G C 2856$}

In Figure 2, we present a montage of the UV, optical, and infrared images of NGC 2856, the northern galaxy in the Arp 285 pair. In the optical images, a dusty spiral pattern and a central bar-like feature are seen in the disk. A series of four clumps are visible along the northern tail in all of the optical images, except for the $u$ image (only two clumps detected)

\footnotetext{
8 IRAF is distributed by the National Optical Astronomy Observatory, which is operated by the Association of Universities for Research in Astronomy, Inc., under cooperative agreement with the National Science Foundation.
} 


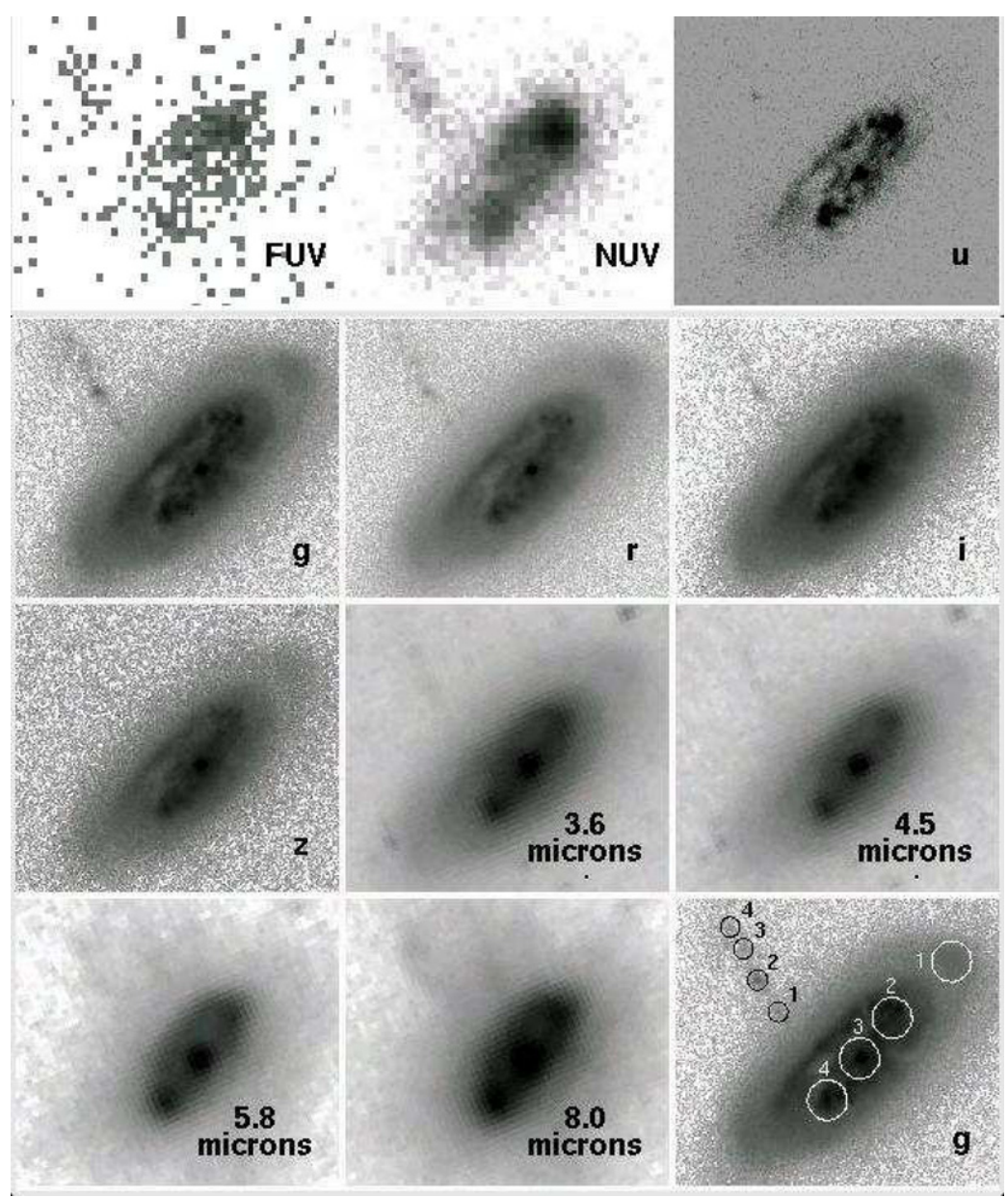

Figure 2. A montage of the GALEX, SDSS, and Spitzer images of NGC 2856, the northern galaxy in Arp 285. North is up and east to the left. The field of view is $1^{\prime} .1 \times 0.9$. Notice the series of clumps in the northern tail. The tail clumps are enclosed by $1^{\prime \prime} .61$ black circles in the last panel on the $g$ image, and are labeled as in Table 2. The NGC 2856 disk clumps listed in Table 2 are marked in the last panel by 4 " radius white circles. Disk clump 1 is the "bright spot" mentioned in Section 3.1 .

and the $z$ image (only one clump detected). These clumps are labeled on the $g$ image in the last panel in Figure 2. From south to north, the separations between the clumps are 7".6 (1.4 kpc), 6.'9 $(1.3 \mathrm{kpc})$, and $5{ }^{\prime \prime} 1(1.0 \mathrm{kpc})$. Clumps 1 and 2 have bright unresolved or marginally-resolved cores (FWHM $<1$ 1.3 = $250 \mathrm{pc}$ ) in the $g$ image; clumps 3 and 4 are fainter in $g$, with multiple peaks.

The Spitzer $5.8 \mu \mathrm{m}$ and $8.0 \mu \mathrm{m}$ images are affected by "banding," where bright point sources (such as galactic nuclei) cause horizontal "bands" in the images (Spitzer Infrared Array Camera Data Manual, Version 2.0, 2005). As discussed in Smith et al. (2007), we corrected for these artifacts by interpolating from nearby clean regions. Unfortunately, the correction was not perfect for NGC 2856, leaving a residual diagonal "stripe" across the rotated image near the tail (see Figure 2). In spite of this, however, clump 3 is detected in all four Spitzer bands, and clump 2 is detected at $3.6 \mu \mathrm{m}$ and $4.5 \mu \mathrm{m}$. Clump 1 is not detected in any of the Spitzer filters, while clump 4 has only a marginal detection at $8.0 \mu \mathrm{m}$. Note that clump 2 is brightest in the SDSS data, but clump 3 is brightest at $8 \mu \mathrm{m}$.

The northern tail is also visible in both the FUV and NUV images. In the longer exposure NUV image, clumps 1-3 are bright, while clump 4 is marginally detected. In the short exposure FUV image, with the low resolution spatial resolution of GALEX the individual clumps are not well resolved. The northern tail is not detected in the 2MASS near-infrared images.

In the SARA H $\alpha$ map (Figure 3), only clump 2 is detected in the northern tail. This implies that clump 3, the brightest clump at $8 \mu \mathrm{m}$, is more extincted. This is consistent with the $\sim 12^{\prime \prime}$ resolution C Array H i map of Chengalur et al. (1994). In this map, two Hi peaks are clearly visible in the northern tail. The brightest $\mathrm{H}_{\mathrm{I}}$ peak is approximately coincident with clump 1, while the second is near clump 3. Thus clump 2 may be less extincted than these other clumps. This is consistent with our analysis of the optical colors (see Section 4.2).

In the $u$ through $4.5 \mu \mathrm{m}$ images of NGC 2856, a "bright spot" is visible in the northwestern edge of the disk of NGC 2856 (see Figure 2). This "bright spot" is also visible in the Arp image (Figure 1), and is marginally detected in the 2MASS $H$ and $K_{s}$ images. However, it is not seen as a discrete source at $5.8 \mu \mathrm{m}, 8.0 \mu \mathrm{m}$, or in the FUV, NUV, $\mathrm{H} \alpha$, or 2MASS $J$ images (Figures 2 and 3). Unlike the clumps in the northern tail, this "bright spot" is smoothly extended in the SDSS images, with an FWHM $\sim 7^{\prime \prime}(1.3 \mathrm{kpc})$ in the $g$ filter, without a compact core or cores.

Within the inner disk of NGC 2856, bright $8 \mu \mathrm{m}$ and $\mathrm{H} \alpha$ sources are visible at the ends of the bar and the nucleus (Figures 2 and 3). The bar is asymmetric, with the clump near 


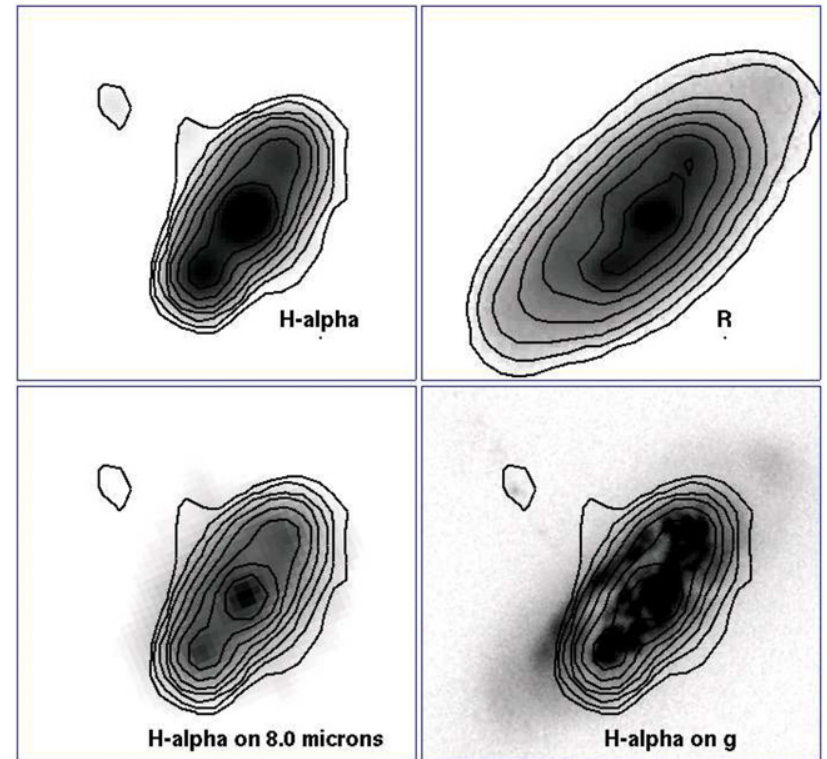

Figure 3. Upper left: the SARA $\mathrm{H} \alpha$ map of NGC 2856. Upper right: the SARA $R$-band map of NGC 2856. Lower left: the SARA H $\alpha$ map of NGC 2856 (contours) superimposed onto the Spitzer $8 \mu \mathrm{m}$ map (gray scale). Lower right: the SARA H $\alpha$ map of NGC 2856 (contours) plotted on the SDSS $g$ map (gray scale). North is up and east to the left. This map has been smoothed by a Gaussian with FWHM $=44^{\prime \prime} .5$. The field of view is $1^{\prime} .0 \times 0.9$.

the southern end of the bar being brighter than the northern source in both $8 \mu \mathrm{m}$ and $\mathrm{H} \alpha$. In the higher-resolution SDSS images, the sources at the ends of the bar are resolved into 2-4 peaks separated by $2^{\prime \prime}-3^{\prime \prime}(0.4-0.6 \mathrm{kpc})$.

In Figure 4, a band-merged approximately true-color optical SDSS image of NGC 2856 is presented. This shows that the clumps in the northern tail are bluer than the main disk of the galaxy. The dust features and the spiral pattern are also visible in this picture. The northeastern spiral arm is bluer than the southwestern portion of the disk. This is also apparent in Figure 2. In the FUV, NUV, and $u$ images, the northeastern portion of the disk is brighter than the southwestern section, but in the longer wavelength images, the disk is more symmetric. This suggests that the difference at shorter wavelengths is due to extinction. This implies that the northeastern side of NGC 2856 is closest to us. This is consistent with the sense of rotation indicated by the H I velocity field (Chengalur et al. 1994), assuming the northwestern spiral arm is trailing.

A connecting bridge between the two galaxies is visible in the smoothed $g$ and $r$ SDSS images (see Figure 5), but is not seen in $u, i$, or $z$. This bridge is aligned with the "bright spot" in the disk. In Figure 5, the northern tail is visible out to $\approx 72^{\prime \prime}$ $(14 \mathrm{kpc})$ from the disk. A bend and a sudden drop-off in brightness is evident in this tail just north of the four bright clumps of star formation. Another possible faint clump is visible in the smoothed $g$ image $\sim 7.9^{\prime \prime}(1.5 \mathrm{kpc})$ northwest of clump 4 , north of the bend. This bend and the bridge are also visible in the Arp image (Figure 1).

\subsection{NGC 2854}

In Figure 6, a montage of the UV, optical, and infrared images of the southern galaxy NGC 2854 is shown. On-going star formation is detected along the spiral arms and at the ends of the bar. The base of the northern tail/bridge appears double

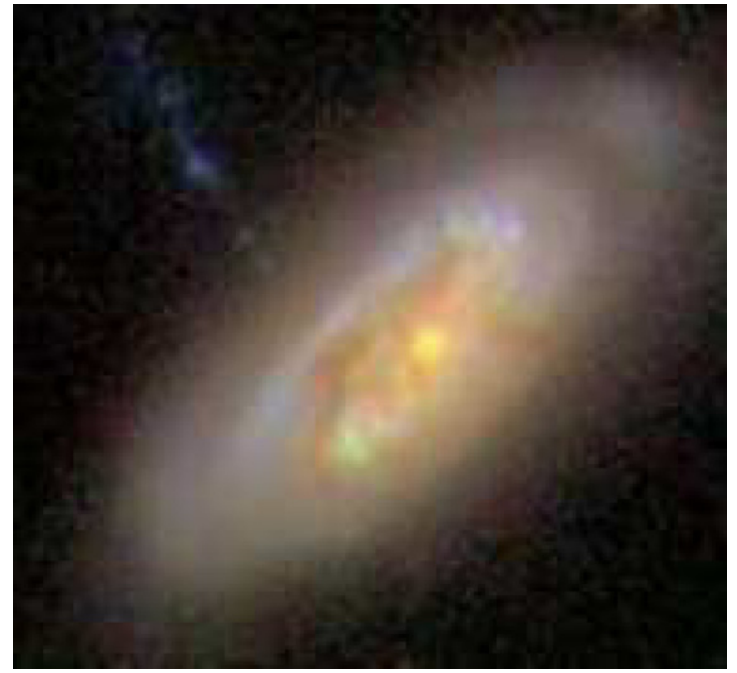

Figure 4. An approximately true-color multi-filter optical SDSS image of NGC 2856. Note that the clumps in the tail are blue, and the northeastern spiral arm is bluer than the southwestern disk. The field of view is $1^{\prime} .1 \times 0^{\prime} .9$.

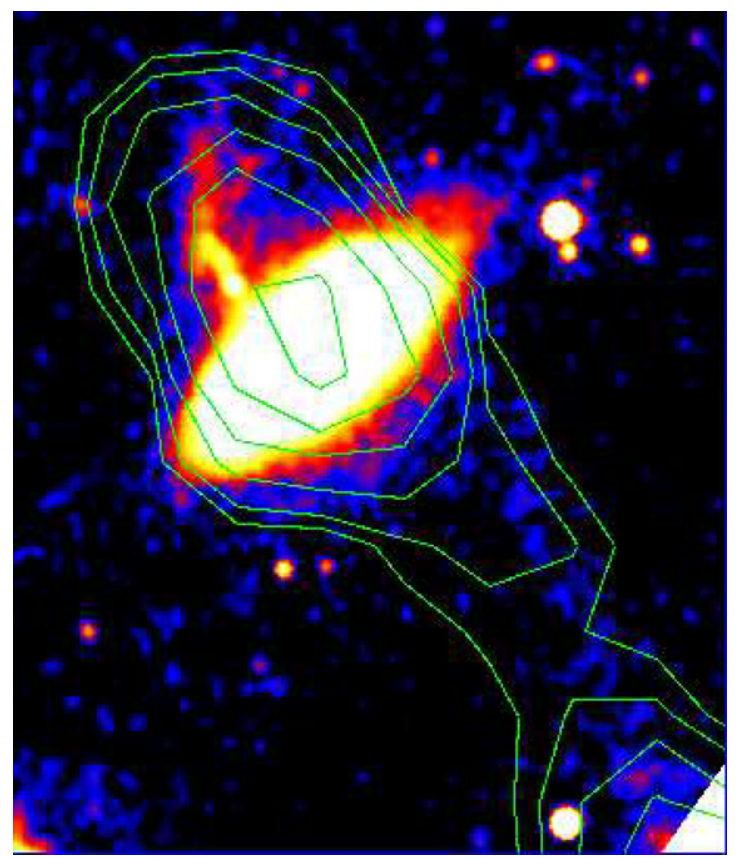

Figure 5. The smoothed SDSS $g$ image of NGC 2856 (color), with $21 \mathrm{~cm}$ H I contours (from Chengalur et al. 1994) superimposed. North is up and east to the left. Notice the bridge connecting this galaxy to its southern companion NGC 2854. Also note the bend in the northern tail north of the clumps marked in Figure 2. The field of view is $3^{\prime} .0 \times 3.3$. The H I beam size is $29^{\prime \prime} \times 29^{\prime \prime}$, and the H I contours are $\left(2.6,4.6,8.1,14,25,43\right.$, and 76) $\times 10^{20} \mathrm{~cm}^{-2}$.

in the $u, g, r, 5.8 \mu \mathrm{m}$, and $8.0 \mu \mathrm{m}$ images. A series of clumps are visible in the spiral arms in both the optical and the infrared images, and $8 \mu \mathrm{m}$-bright sources are seen at the ends of the bar. For some clumps, there are $1^{\prime \prime}-2^{\prime \prime}$ offsets between the optical and $8 \mu \mathrm{m}$ peaks; for others, including the nuclear source, there is no clear optical peak associated with the $8 \mu \mathrm{m}$ source. In the last panel of Figure 6, we identify eight clumps selected based on the $8 \mu \mathrm{m}$ image.

The SARA H $\alpha$ and R maps of NGC 2854 are presented in Figure 7, with the $\mathrm{H} \alpha$ superimposed on the $g$ and $8 \mu \mathrm{m}$ images. 

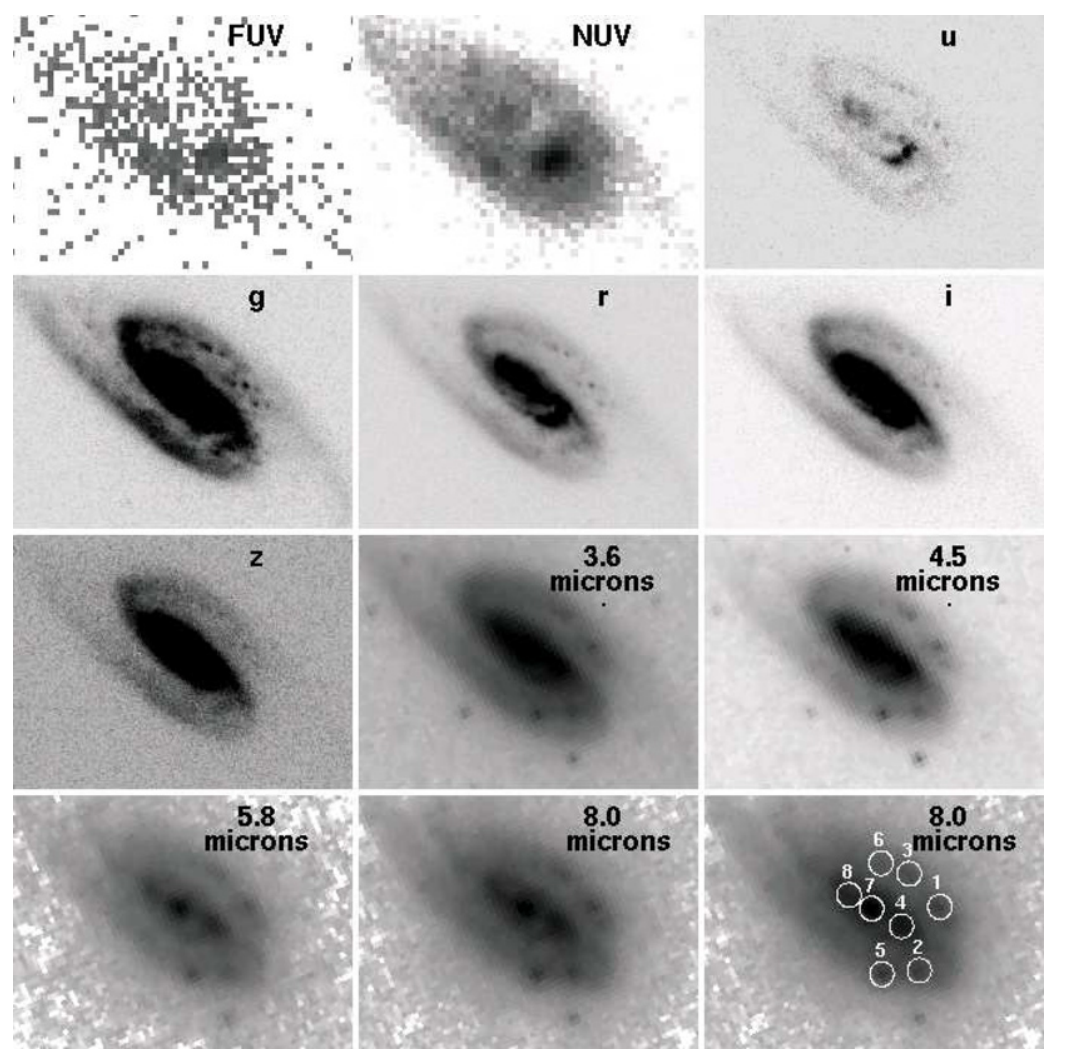

Figure 6. A montage of images of the southern galaxy in Arp 285, NGC 2854. North is up and east to the left. The field of view is $1^{\prime} .2 \times 1^{\prime} .0$. Notice the series of clumps in the northern spiral arm. In the UV and optical, the southern end of the bar is brighter than the northern end. At longer wavelengths, the disk is more symmetrical. The positions of the $8 \mu \mathrm{m}$-selected clumps in Table 2 are circled on the $8 \mu \mathrm{m}$ image in the last panel. The circles have 2 '. 8 radii.

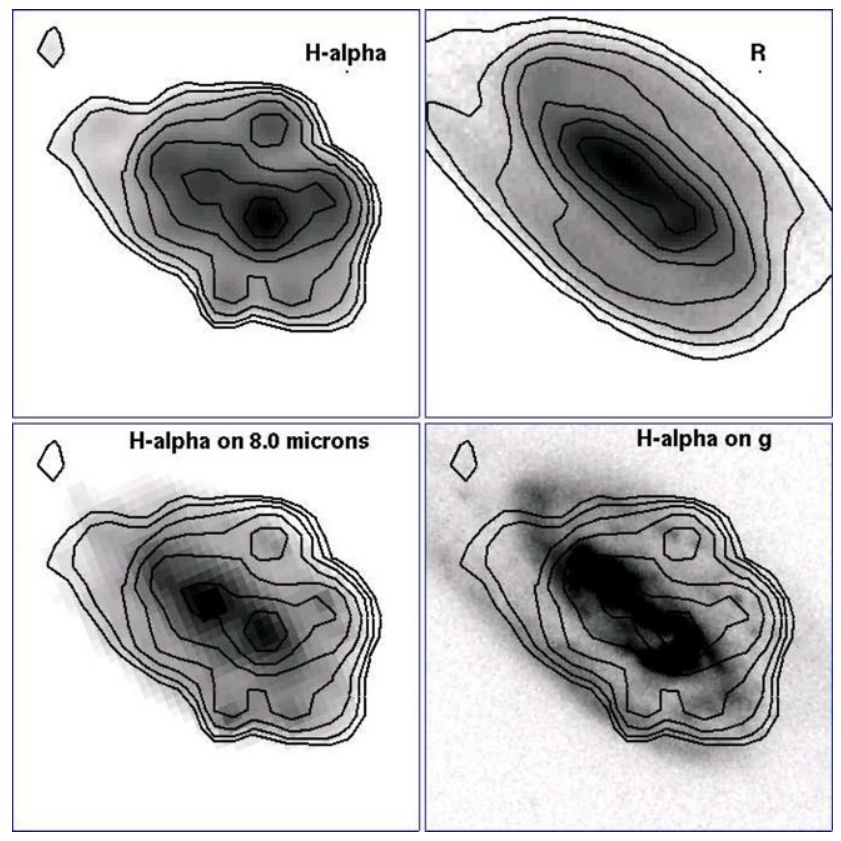

Figure 7. Upper left: the SARA H $\alpha$ map of NGC 2854. Upper right: the SARA $R$-band map of NGC 2854. Lower left: the SARA H $\alpha$ map of NGC 2854 (contours) superimposed onto the Spitzer $8 \mu \mathrm{m}$ map (gray scale). Lower right: the SARA H $\alpha$ map of NGC 2854 (contours) plotted on the SDSS $g$ map (gray scale). North is up and east to the left. This map has been smoothed by a Gaussian with FWHM $=4{ }^{\prime \prime} .5$. The field of view is $1.1 \times 1$ 1.1.

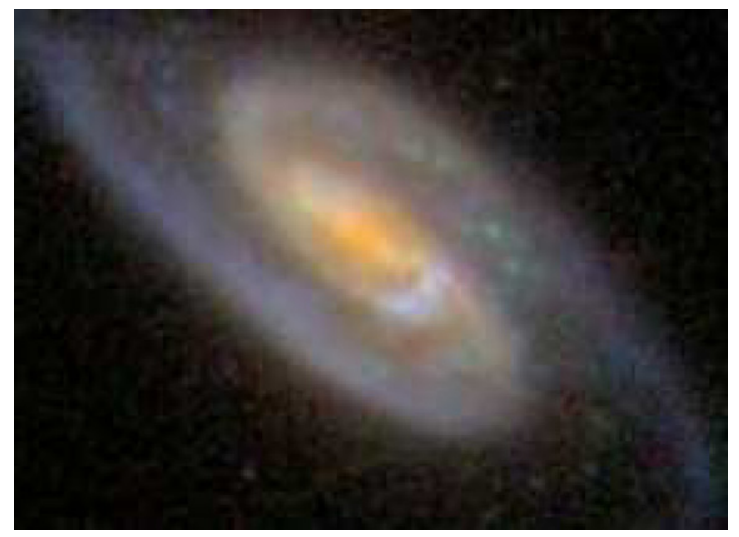

Figure 8. An approximately true-color multi-filter optical SDSS image of NGC 2854. North is up and east to the left. Note that the clumps in the northern arm are blue, and the southeastern end of the bar is bluer than the northern end. The southern arm/tail is also bluer than that in the north. The field of view is $1^{\prime} \cdot 2 \times 11^{\prime} 0$.

All of the $8 \mu \mathrm{m}$ clumps except clump 6 were detected in $\mathrm{H} \alpha$. In addition, possible $\mathrm{H} \alpha$ emission is seen associated with the western portion of the double bridge.

An approximately true-color optical SDSS image of NGC 2854 is displayed in Figure 8. The double bridge structure is visible in this image. The knots along the northern arm are visible. The southern end of the bar is bluer than the northern 


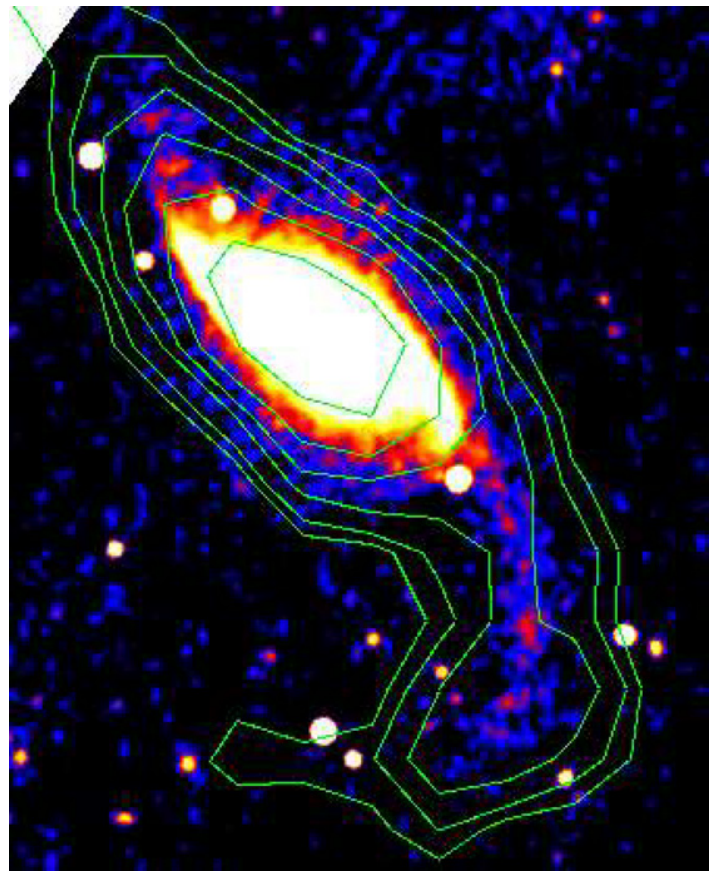

Figure 9. The smoothed $g$ image of NGC 2854, with $21 \mathrm{~cm} \mathrm{H}$ I contours from Chengalur et al. (1994) superimposed. North is up and east to the left. The field of view is $3^{\prime} .4 \times 4^{\prime} .2$. Note the long tidal tail extending $1^{\prime} .8$ to the south. The $\mathrm{H}$ beamsize is $29^{\prime \prime} \times 29^{\prime \prime}$, and the H I contours are $(2.6,4.6,8.1,14,25,43$, and 76) $\times 10^{20} \mathrm{~cm}^{-2}$.

end, and the southern arm/tail is bluer than the northern arm. The southwestern end of the bar is particularly bright in the UV, but less so in the mid-infrared (Figure 6). NGC 2854 appears more symmetric in the Spitzer images than at shorter wavelengths. This implies that the color variations seen in the optical/UV are due to extinction. The color variations suggest that the southern side of NGC 2854 is the near side, consistent with the $\mathrm{HI}$ velocity field of Chengalur et al. (1994) and trailing spiral arms.

The smoothed $g$ image of NGC 2854 is presented in Figure 9. A faint optical tail is detected, extending 1.8 (20 kpc) to the south, coincident with the long $\mathrm{H}$ I tail seen by Chengalur et al. (1994). This tail is also visible in the smoothed FUV and NUV images.

Approximately $3^{\prime}$ due north of NGC 2854 , at $9^{\mathrm{h}} 24^{\mathrm{m}} 2.9^{\mathrm{s}}$, $49^{\circ} 14^{\prime} 41^{\prime \prime}$ (J2000), a small angular size galaxy is visible on the Arp image (Figure 1). This galaxy is detected in all of the GALEX and SDSS bands, as well as the Spitzer $3.6 \mu \mathrm{m}$ and $4.5 \mu \mathrm{m}$ filters. At the present time, no redshift is available for this source, so it is unknown whether it is associated with Arp 285. Its proximity to a very bright star (see Figure 1) prevents a reliable $\mathrm{H} \alpha$ detection. The magnitudes of this galaxy in the various filters are given in Table 1. The UV/optical colors are quite blue, consistent with a young stellar population.

\section{CLUMP ANALYSIS}

\subsection{Photometry}

The positions, SDSS, and GALEX magnitudes and Spitzer flux densities of the clumps in the NGC 2856 tail and the two disks of Arp 285 are given in Table 2 in R.A. order. The photometry was done using the IRAF daophot routine. For the tail and the "bright spot," the positions were determined by eye, based on the $g$ image, while the disk positions are the $8 \mu \mathrm{m}$ peaks. These positions are marked in the last panels of Figures 2 and 6. The apertures we utilized are given in Table 3, along with the aperture corrections used to correct to total magnitudes. Since the clumps in the northern tail are separated by only $\sim 5^{\prime \prime}-7^{\prime \prime}$, we used relatively small apertures for the tail clump photometry. For the disk clumps, we used larger apertures because of less crowding, and because there are sometimes multiple optical peaks associated with a single Spitzer source.

For background subtraction, the local galaxian background was determined using the mode in an annulus surrounding the source (see Table 3 ). To estimate the uncertainty in the colors of the clumps due to background subtraction, in addition to the statistical uncertainties determined from the root mean square (rms) in the background annuli, in calculating the colors we added in quadrature a second uncertainty term, determined from comparing the clump colors obtained with the above method with those obtained with a slightly larger annulus.

We also extracted approximate $J, H$, and $K_{s}$ photometry for the clumps from the 2MASS Atlas images. These near-infrared fluxes were not used in the population synthesis modeling (Section 4.2), but were only used for comparison with the Spitzer data (Section 4.3); thus we did not include the second sky annulus.

We also obtained the total FUV and NUV fluxes for a $25^{\prime \prime} .8 \times$ 9.7 region containing the four knots. These magnitudes are also given in Table 2, along with the SDSS and Spitzer magnitudes for the same region. The uncertainties given in Table 2 for this region were calculated as in Smith et al. (2007), including both statistical uncertainties and the uncertainty in the sky level. The total $g$ flux in this rectangular region is $\sim 3 \times$ the sum of the $g$ fluxes for the four clumps (see Table 2); thus there is significant diffuse emission in this tail.

\subsection{Ages}

In Figure 10, we plot the SDSS $g-r$ colors for both the tail and disk clumps versus their $u-g$ colors. In Figure 11, NUV $-g$ is plotted against $g-r$. Similar plots of $r-i$ versus $g-r, i-z$ versus $r-i$, and FUV-NUV versus $g-r$ are presented in the Appendix. These figures also include the colors for the $25^{\prime \prime} .8 \times$ 9 9.7 rectangular region that includes the four clumps in the northern tail.

To estimate the ages of these clumps, we calculated theoretical colors for star-forming regions using version 5.1 of the Starburst99 population synthesis code (Leitherer et al. 1999). This version includes the Padova asymptotic giant-branch stellar models (Vázquez \& Leitherer 2005). These models assume an instantaneous burst with a Kroupa (2002) initial mass function (IMF) and an initial mass range of $0.1-100 M_{\odot}$. We calculated colors for a range of ages $\tau$ from $1 \mathrm{Myr}$ to $10 \mathrm{Gyr}$. We used a time step size of $\Delta \tau=1 \mathrm{Myr}$ for $0<\tau<1 \mathrm{Gyr}$, and $\Delta \tau=100$ Myr for $1.1<\tau<10$ Gyr. The Calzetti et al. (1994) starburst dust-reddening law was assumed. We also generated models using a Salpeter IMF, and found the colors differed only slightly from those with the Kroupa IMF. This is consistent with earlier studies (MacArthur et al. 2004). To the broadband fluxes, we added in the contributions from $\mathrm{H} \alpha$ emission, which can be substantial in the $r$ filter. For a 1 Myr star-forming region, $\mathrm{H} \alpha$ decreases the $r$ magnitude by $1.1 \mathrm{mag}$; at $5 \mathrm{Myr}, \mathrm{H} \alpha$ contributes $\sim 0.25$ mag (see Figure 10).

To systematically estimate ages and extinctions for the clumps in Table 2, we used a $\chi^{2}$ minimization calculation (e.g., Pasquali et al. 2003) to determine the fit of the observed colors to that of 
Table 2

Magnitudes and Flux Densities for Clumps in Arp 285

\begin{tabular}{|c|c|c|c|c|c|c|c|c|c|c|c|c|c|}
\hline ID & $\begin{array}{c}\text { R.A. } \\
\text { (J2000) }\end{array}$ & $\begin{array}{c}\text { Decl. } \\
(\mathrm{J} 2000)\end{array}$ & $\begin{array}{l}\text { FUV } \\
\text { (mag) }\end{array}$ & $\begin{array}{l}\text { NUV } \\
(\mathrm{mag})\end{array}$ & $\begin{array}{c}u \\
(\mathrm{mag})\end{array}$ & $\begin{array}{c}g \\
(\mathrm{mag})\end{array}$ & $\begin{array}{c}r \\
(\mathrm{mag})\end{array}$ & $\begin{array}{c}i \\
(\mathrm{mag})\end{array}$ & $\begin{array}{c}z \\
(\mathrm{mag})\end{array}$ & $\begin{array}{c}F_{3.6 \mu \mathrm{m}} \\
(\mathrm{mJy})\end{array}$ & $\begin{array}{c}F_{4.5 \mu \mathrm{m}} \\
(\mathrm{mJy})\end{array}$ & $\begin{array}{c}F_{5.8 \mu \mathrm{m}} \\
(\mathrm{mJy})\end{array}$ & $\begin{array}{c}F_{8.0 \mu \mathrm{m}} \\
(\mathrm{mJy})\end{array}$ \\
\hline \multicolumn{14}{|c|}{ Clumps in the Northern NGC 2856 Tail } \\
\hline 1 & 92417.7 & 49156.2 & $\ldots$ & $22.7 \pm 0.3$ & $\geqslant 22.18$ & $21.74 \pm 0.20$ & $21.36 \pm 0.22$ & $20.98 \pm 0.25$ & $\geqslant 20.45$ & $\leqslant 0.084$ & $\leqslant 0.059$ & $\leqslant 0.14$ & $\leqslant 0.47$ \\
\hline 2 & 92418.2 & 491512.5 & $\ldots$ & $21.2 \pm 0.1$ & $20.87 \pm 0.09$ & $20.73 \pm 0.04$ & $20.69 \pm 0.06$ & $20.87 \pm 0.11$ & $20.72 \pm 0.34$ & $0.017 \pm 0.004$ & $0.013 \pm 0.004$ & $\leqslant 0.05$ & $\leqslant 0.13$ \\
\hline 3 & 92418.5 & 491518.7 & $\ldots$ & $21.3 \pm 0.1$ & $21.44 \pm 0.22$ & $21.56 \pm 0.12$ & $21.37 \pm 0.11$ & $21.88 \pm 0.23$ & $\geqslant 20.87$ & $0.014 \pm 0.002$ & $0.012 \pm 0.002$ & $0.08 \pm 0.01$ & $0.25 \pm 0.03$ \\
\hline 4 & 92418.7 & 491522.9 & $\ldots$ & $22.0 \pm 0.1$ & $\geqslant 22.24$ & $22.16 \pm 0.10$ & $22.19 \pm 0.18$ & $22.27 \pm 0.28$ & $\geqslant 20.89$ & $\leqslant 0.006$ & $\leqslant 0.005$ & $\leqslant 0.03$ & $0.10 \pm 0.03$ \\
\hline \multicolumn{14}{|c|}{$25^{\prime \prime} .8 \times 9$ 9.7 Region Including Clumps in Northern Tail ${ }^{\mathrm{b}}$} \\
\hline \multicolumn{14}{|c|}{ Clumps in the NGC 2856 disk } \\
\hline $1^{\mathrm{c}}$ & 92414.2 & 491516.1 & $\geqslant 21.38$ & $\geqslant 22.98$ & $20.32 \pm 0.05$ & $18.97 \pm 0.01$ & $18.39 \pm 0.01$ & $17.99 \pm 0.01$ & $18.20 \pm 0.02$ & $0.260 \pm 0.051$ & $0.162 \pm 0.032$ & $\leqslant 0.26$ & $\leqslant 0.77$ \\
\hline 2 & 92415.4 & 49154.9 & $18.68 \pm 0.11$ & $18.78 \pm 0.07$ & $17.40 \pm 0.01$ & $16.57 \pm 0.01$ & $15.94 \pm 0.01$ & $15.64 \pm 0.01$ & $15.30 \pm 0.01$ & $5.008 \pm 1.000$ & $3.397 \pm 0.730$ & $13.74 \pm 2.70$ & $43.83 \pm 7.80$ \\
\hline $3^{\mathrm{d}}$ & 92416.1 & 491457.0 & $\geqslant 19.86$ & $\geqslant 21.00$ & $18.32 \pm 0.01$ & $16.58 \pm 0.01$ & $15.38 \pm 0.01$ & $14.93 \pm 0.01$ & $14.50 \pm 0.01$ & $14.085 \pm 0.421$ & $10.451 \pm 0.289$ & $42.38 \pm 1.19$ & $135.01 \pm 3.51$ \\
\hline 4 & 92416.7 & 491448.7 & $20.17 \pm 0.18$ & $19.76 \pm 0.08$ & $18.06 \pm 0.01$ & $16.63 \pm 0.01$ & $15.93 \pm 0.01$ & $15.73 \pm 0.01$ & $15.36 \pm 0.02$ & $6.178 \pm 1.005$ & $4.325 \pm 0.733$ & $19.14 \pm 2.73$ & $61.65 \pm 7.91$ \\
\hline \multicolumn{14}{|c|}{ Clumps in the NGC 2854 disk } \\
\hline 1 & 9241.5 & 491215.5 & $\geqslant 19.82$ & $20.79 \pm 0.31$ & $20.44 \pm 0.09$ & $19.67 \pm 0.09$ & $18.98 \pm 0.08$ & $18.80 \pm 0.10$ & $18.87 \pm 0.11$ & $\leqslant 0.847$ & $\leqslant 0.548$ & $1.94 \pm 0.58$ & $5.79 \pm 1.66$ \\
\hline 2 & 9242.0 & 49121.1 & $\geqslant 19.66$ & $\geqslant 20.28$ & $20.43 \pm 0.07$ & $19.41 \pm 0.06$ & $18.79 \pm 0.06$ & $18.49 \pm 0.06$ & $17.96 \pm 0.06$ & $0.953 \pm 0.234$ & $0.591 \pm 0.150$ & $2.31 \pm 0.51$ & $7.21 \pm 1.42$ \\
\hline 3 & 9242.3 & 491223.1 & $\geqslant 20.67$ & $\geqslant 21.45$ & $20.78 \pm 0.07$ & $20.32 \pm 0.04$ & $19.41 \pm 0.05$ & $19.50 \pm 0.06$ & $19.65 \pm 0.07$ & $\leqslant 1.602$ & $\leqslant 1.074$ & $\leqslant 3.28$ & $\leqslant 8.47$ \\
\hline 4 & 9242.4 & 491211.2 & $19.37 \pm 0.24$ & $19.03 \pm 0.15$ & $17.94 \pm 0.02$ & $16.86 \pm 0.02$ & $16.14 \pm 0.02$ & $15.84 \pm 0.03$ & $15.55 \pm 0.04$ & $4.169 \pm 0.556$ & $2.683 \pm 0.377$ & $8.49 \pm 1.08$ & $25.85 \pm 2.98$ \\
\hline 5 & 9242.9 & 49120.3 & $\geqslant 19.83$ & $\geqslant 20.38$ & $20.01 \pm 0.08$ & $19.29 \pm 0.06$ & $18.83 \pm 0.06$ & $18.94 \pm 0.06$ & $18.69 \pm 0.06$ & $0.833 \pm 0.254$ & $0.570 \pm 0.160$ & $2.21 \pm 0.48$ & $6.80 \pm 1.43$ \\
\hline 6 & 9242.9 & 491225.5 & $20.54 \pm 0.34$ & $\geqslant 21.52$ & $20.52 \pm 0.10$ & $19.85 \pm 0.11$ & $19.43 \pm 0.13$ & $19.19 \pm 0.13$ & $18.89 \pm 0.15$ & $\leqslant 1.815$ & $\leqslant 1.218$ & $\leqslant 3.46$ & $\leqslant 9.51$ \\
\hline $7^{\mathrm{d}}$ & 9243.1 & 491215.2 & $\geqslant 19.84$ & $\geqslant 21.00$ & $19.47 \pm 0.06$ & $17.51 \pm 0.03$ & $16.21 \pm 0.02$ & $15.53 \pm 0.02$ & $15.04 \pm 0.01$ & $8.881 \pm 0.257$ & $5.927 \pm 0.166$ & $17.15 \pm 0.50$ & $50.58 \pm 1.40$ \\
\hline 8 & 9243.7 & 491218.3 & $20.22 \pm 0.34$ & $20.55 \pm 0.11$ & $18.63 \pm 0.03$ & $16.99 \pm 0.02$ & $16.22 \pm 0.03$ & $15.86 \pm 0.04$ & $15.59 \pm 0.04$ & $4.019 \pm 0.451$ & $2.560 \pm 0.300$ & $6.28 \pm 0.92$ & $20.53 \pm 1.93$ \\
\hline
\end{tabular}

Notes.

a Except where noted, only statistical uncertainties are included, calculated from the rms in the smaller sky annulus used.

b Uncertainties include both statistical uncertainties and uncertainties due to sky subtraction, calculated as by Smith et al. (2007).

c "Bright spot" in the northwestern edge of the disk.

d Nucleus. 
Table 3

Parameters for Clump Photometry for Arp 285

\begin{tabular}{|c|c|c|c|c|}
\hline Telescope & $\begin{array}{l}\text { Aperture } \\
\text { radius }\end{array}$ & $\begin{array}{l}\text { Aperture } \\
\text { correction } \\
(\mathrm{mag})\end{array}$ & $\begin{array}{c}\text { Inner } \\
\text { sky } \\
\text { annulus }\end{array}$ & $\begin{array}{c}\text { Outer } \\
\text { sky } \\
\text { annulus }\end{array}$ \\
\hline \multicolumn{5}{|c|}{ Clumps in the Northern NGC 2856 tail } \\
\hline GALEX & $2 \operatorname{pix}(3.0)$ & $0.45 / 0$ & $2-5$ pix ( & $4-7$ pix $\left(6^{\prime \prime}-100^{\prime} 5\right)$ \\
\hline DSS & 5 pix $\left(22^{\prime \prime} 0\right)$ & $0.08^{\mathrm{b}}$ & $10-20$ pix $\left(4^{\prime \prime}-8^{\prime \prime}\right)$ & $15-30$ pix $\left(6^{\prime \prime}-12^{\prime \prime}\right)$ \\
\hline pitzer & 2 pix (2". 4$)$ & $0.21,0.23,0.35,0.50^{\mathrm{c}}$ & $2-8$ pix $\left(22^{\prime \prime} 4-9 !^{\prime \prime} 6\right)$ & $5-9$ pix $\left(6^{\prime \prime}-10^{\prime} 8\right)$ \\
\hline MASS & 3 pix $\left(33^{\prime \prime} 0\right)$ & $0.1^{\mathrm{b}}$ & 4-7 pix (4.". $\left.0-7{ }^{\prime \prime} .0\right)$ & \\
\hline \multicolumn{5}{|c|}{ Clumps in the NGC 2856 disk } \\
\hline FLLEX & 2 pix (3..0) & $0.45 / 0.69^{\mathrm{a}}$ & $2-5$ pix $\left(3^{\prime \prime}-7^{\prime \prime} .5\right)$ & $4-7$ pix $\left(6^{\prime \prime}-10^{\prime \prime} 5\right)$ \\
\hline DSS & 10 pix $\left(4^{\prime \prime}\right)$ & $0.03^{\mathrm{b}}$ & $10-20$ pix $\left(4^{\prime \prime}-8^{\prime \prime}\right)$ & $15-30$ pix $\left(6^{\prime \prime}-12^{\prime \prime}\right)$ \\
\hline pitzer & 3 pix (3". 6$)$ & $0.13,0.13,0.15,0.23^{\mathrm{c}}$ & $5-10$ pix $\left(6^{\prime \prime}-12^{\prime \prime}\right)$ & $9-12$ pix $\left(10^{\prime \prime} 8-14^{\prime \prime} 4\right)$ \\
\hline MASS & 3 pix $(3 . .0)$ & $0.1^{\mathrm{b}}$ & $4-7$ pix $\left(4^{\prime \prime} 0-7.0\right)$ & \\
\hline \multicolumn{5}{|c|}{ the NGC 2854 disk } \\
\hline GALEX & $2(3 \prime$. & $0.45 / 0.69^{\mathrm{a}}$ & $2-5$ pix $\left(3^{\prime \prime}-7 " .5\right)$ & $4-7$ pix $\left(6^{\prime \prime}-10^{\prime \prime} 5\right)$ \\
\hline & $7 \mathrm{pi}$ & $0.03^{\mathrm{b}}$ & $10-20$ pix $\left(4^{\prime \prime}-8^{\prime \prime}\right)$ & $15-30$ pix $\left(6^{\prime \prime}-12^{\prime \prime}\right)$ \\
\hline Spitze & 3 pi & $0.13,0.13,0.15,0.23^{\mathrm{c}}$ & $5-10$ pix $\left(6^{\prime \prime}-12^{\prime \prime}\right)$ & $9-12$ pix $\left(10^{\prime \prime} 8-144^{\prime \prime} 4\right)$ \\
\hline 2MASS & 3 pix $\left(3^{\prime \prime} 0\right)$ & $0.1^{\mathrm{b}}$ & $4-7$ pix $\left(4 ! .0-77^{\prime \prime} 0\right)$ & \\
\hline
\end{tabular}

Notes.

${ }^{a}$ For FUV and NUV, respectively. NUV from bright stars in field. FUV from bright stars in the Arp 65 field.

${ }^{\mathrm{b}}$ From bright stars in the field.

${ }^{\mathrm{c}}$ For 3.6, 4.5, 5.8, and $8.0 \mu \mathrm{m}$, respectively, from the IRAC Data Manual, Version 3.

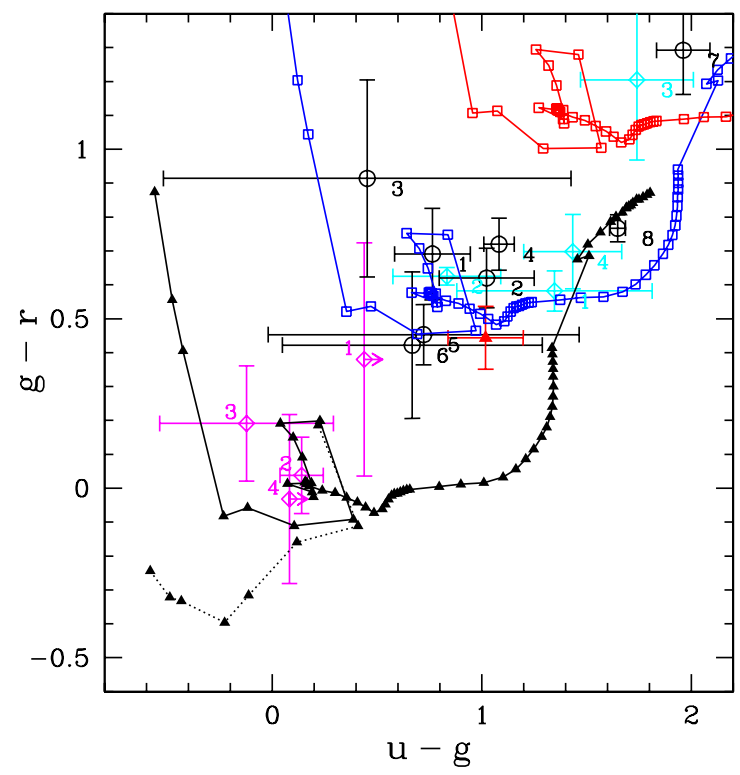

Figure 10. The $g-r$ vs. $u-g$ colors of the clumps in the NGC 2856 tail (magenta open diamonds), the NGC 2856 disk (cyan open diamonds), and the NGC 2854 disk (black open circles). The clumps are identified by their numbers in Table 2, labeled in the same color. These data are compared with solar metallicity Kroupa IMF instantaneous burst population synthesis models with extinction of $E(B-V)=0$ (black filled triangles), 0.5 (blue open squares), and 1.0 (red open squares). To show the effect of $\mathrm{H} \alpha$ on the $g-r$ color, for the zero extinction model two curves are shown: with (solid line) and without (dotted line) $\mathrm{H} \alpha$. The model ages start with an age of $1 \mathrm{Myr}$ for the point on the left end of the curve, increase by $1 \mathrm{Myr}$ steps to $20 \mathrm{Myr}$, then by $5 \mathrm{Myr}$ steps to $50 \mathrm{Myr}, 10 \mathrm{Myr}$ steps to $100 \mathrm{Myr}, 100 \mathrm{Myr}$ steps to $1 \mathrm{Gyr}$, and $500 \mathrm{Myr}$ steps to 10 Gyr. The red filled triangle shows the colors of the $25^{\prime \prime} .6 \times 9$ 9'7 region enclosing all four knots in the northern tail.

the models:

$$
\chi^{2}=\sum_{i=1}^{N}\left(\frac{\mathrm{obs}_{i}-\operatorname{model}_{i}}{\sigma_{i}}\right)^{2} .
$$

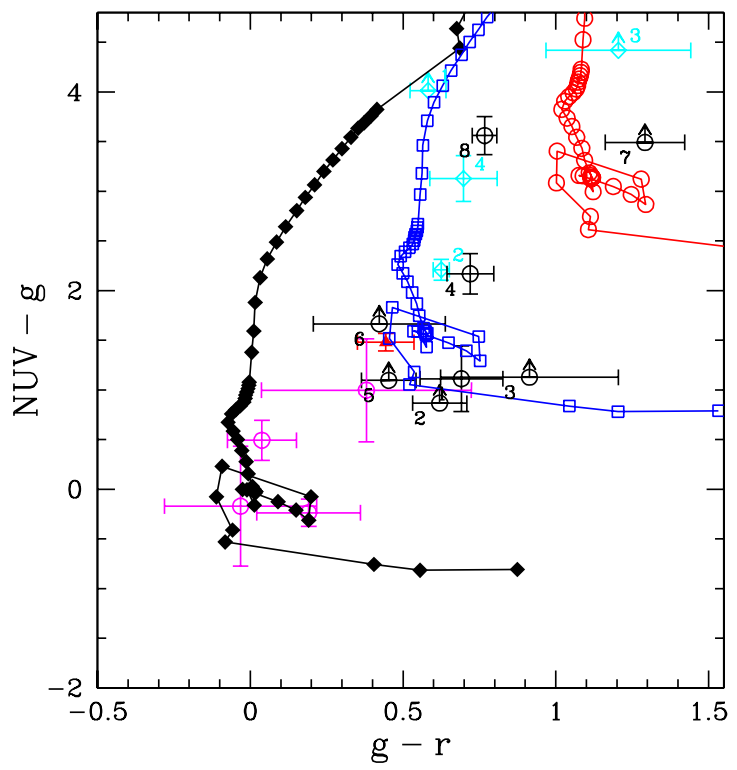

Figure 11. The NUV $-g$ color of the $25^{\prime \prime} 6 \times 9$ 9.7 region in the northern tail, plotted against $g-r$ (red filled triangle). The clumps in the tail (magenta open diamonds), the NGC 2856 disk (cyan open diamonds), and the NGC 2854 disk (black open circles) are also plotted. Solar metallicity Kroupa IMF instantaneous burst population synthesis model colors are also shown, with extinction of $E(B-V)=0$ (black diamonds), 0.5 (blue open squares), and 1.0 (red open circles). The model ages start with an age of $1 \mathrm{Myr}$ for the point at lower left end of the curve, increased by 1 Myr steps to $20 \mathrm{Myr}$, then by $5 \mathrm{Myr}$ steps to $50 \mathrm{Myr}$, then $10 \mathrm{Myr}$ steps to $100 \mathrm{Myr}, 100 \mathrm{Myr}$ steps to $1 \mathrm{Gyr}$, and $500 \mathrm{Myr}$ steps to $10 \mathrm{Gyr}$. All models include $\mathrm{H} \alpha$.

In this equation, $N$ is the number of colors used in the analysis, $\mathrm{obs}_{i}$ is the observed color, model ${ }_{i}$ is the corresponding model color, and $\sigma_{i}$ is the uncertainty in the obs ${ }_{i}$ color. A good fit is indicated by $\chi^{2}<N$. In these calculations, we did not include filters with non-detections. In a few cases, it was not possible 
Table 4

Model Ages and Extinctions for Clumps with Solar Metallicity Models ${ }^{\mathrm{a}}$

\begin{tabular}{|c|c|c|c|}
\hline Clump & $\begin{array}{c}\text { Age } \\
(\mathrm{Myr})\end{array}$ & $\begin{array}{c}E(B-V) \\
(\mathrm{mag})\end{array}$ & Colors used \\
\hline \multicolumn{4}{|c|}{ NGC 2856 tail clumps } \\
\hline 1 & $18 \pm{ }_{15}^{149}$ & $0.3 \pm 0.3$ & $\mathrm{NUV}-g, g-r, r-i$ \\
\hline 2 & $7 \pm{ }_{2}^{42}$ & $0.1 \pm 0.1$ & $\mathrm{NUV}-g, u-g, g-r, r-i, i-z$ \\
\hline 3 & $4 \pm \frac{2}{1}$ & $0.1 \pm 0.1$ & $\mathrm{NUV}-g, u-g, g-r, r-i$ \\
\hline 4 & $15 \pm{ }_{12}^{8}$ & $0.0 \pm 0.2$ & $\mathrm{NUV}-g, g-r, r-i$ \\
\hline \multicolumn{4}{|c|}{ NGC 2856 disk clumps } \\
\hline $1^{\mathrm{b}}$ & $142 \pm{ }_{137}^{1358}$ & $0.5 \pm 0.5$ & $u-g, g-r, r-i$ \\
\hline 2 & $6 \pm \frac{2}{1}$ & $0.68 \pm{ }_{0.1}^{0.04}$ & $\mathrm{NUV}-g, u-g, g-r, r-i, i-z$ \\
\hline $3^{\mathrm{c}}$ & $6900 \pm 6800$ & $0.2 \pm \begin{array}{l}0.7 \\
0.1\end{array}$ & $u-g, g-r, r-i, i-z$ \\
\hline 4 & $88 \pm{ }_{38}^{281}$ & $0.6 \pm_{0.3}^{0.1}$ & $\mathrm{NUV}-g, u-g, g-r, r-i, i-z$ \\
\hline \multicolumn{4}{|c|}{ NGC 2854 disk clumps } \\
\hline 1 & $8 \pm \frac{4}{5}$ & $0.4 \pm{ }_{0.2}^{0.4}$ & $\mathrm{NUV}-g, u-g, g-r, r-i, i-z$ \\
\hline 2 & $7 \pm \frac{2393}{2}$ & $0.6 \pm 0.2$ & $u-g, g-r, r-i, i-z$ \\
\hline 3 & $4 \pm \frac{5}{3}$ & $0.7 \pm 0.4$ & $u-g, g-r, r-i, i-z$ \\
\hline 4 & $7 \pm \frac{1}{2}$ & $0.6 \pm 0.2$ & $u-g, g-r, r-i$ \\
\hline 5 & $6 \pm \pm_{2}^{1394}$ & $0.5 \pm 0.2$ & $u-g, g-r, r-i, i-z$ \\
\hline 6 & $36 \pm{ }_{32}^{3164}$ & $0.4 \pm_{0.3}^{0.5}$ & $u-g, g-r, r-i, i-z$ \\
\hline $7^{\mathrm{c}}$ & $7 \pm{ }_{2}^{7893}$ & $1.3 \pm{ }_{1.0}^{0.1}$ & $u-g, g-r, r-i$ \\
\hline 8 & $1200 \pm \underset{259}{5500}$ & $0.2 \pm \begin{array}{l}0.1 \\
0.2\end{array}$ & $u-g, g-r, r-i, i-z$ \\
\hline
\end{tabular}

Notes.

a All clump ages obtained with instantaneous burst models. The oldest models run were $10 \mathrm{Gyr}$ old.

b "Bright spot" in the northwestern edge of the disk. The upper limit on the NUV flux further constrains this age to $\gtrsim 400 \mathrm{Myr}$ (see Figure 14 and Section 4.2).

${ }^{\mathrm{c}}$ Nucleus.

to find a good fit when the low-signal-to-noise ratio (S/N) FUV or $z$ fluxes were included. In these cases, they were not used in determining the ages.

To estimate the uncertainties in the best-fitted parameters, we used the $\Delta \chi^{2}$ method (Press et al. 1992) to determine $68.3 \%$ confidence levels for the parameters. The best-fitted parameters and their uncertainties are given in Table 4, along with the colors used in the fits. We assumed solar metallicity in these models. In the Appendix, we give results assuming 1/5 solar metallicity. The derived age ranges for the two metallicities are similar; thus the assumed metallicity has little effect on the derived ages. We note that, because the clump masses are relatively low (see Section 4.4), stochastic sampling of the IMF can affect the age determinations (e.g., Cerviño et al. 2002). We do not include this effect in our calculations.

On the color-color plots of the clump colors, we superimpose the solar metallicity models. Using only optical colors, it is often difficult to distinguish between reddening due to age and reddening due to extinction (see Figure 10). Fortunately, however, some clumps were detected in the UV, which in some cases can constrain the ages further (see Table 4). The clumps in the tail have very blue optical/UV colors; thus they have both low extinctions and young ages, with $E(B-V) \sim 0.1$ and ages $\sim 4-20$ Myr (Table 4). Clump 1 is slightly redder than the other clumps, implying a slightly older age and/or a higher extinction.

As noted previously, in the H I map of Chengalur et al. (1994), two peaks are visible in the tail, near clumps 1 and 3 . The brightest peak has an $\mathrm{H}$ I column density $\approx 2 \times 10^{21}$ atoms $\mathrm{cm}^{-2}$, while the second has $N(\mathrm{HI}) \approx 10^{21}$ atoms $\mathrm{cm}^{-2}$. Assuming the standard Galactic $N(\mathrm{H})$-to-extinction ratio of $N(\mathrm{H}) / E(B-V)=$ $5.8 \times 10^{21}$ atoms cm$~^{-2} \mathrm{mag}^{-1}$ (Bohlin et al. 1978) and neglecting possible molecular gas, these imply $E(B-V) \approx$ 0.3 to clump 1 and $E(B-V) \approx 0.15$ to clump 3 . These are consistent with the population synthesis results (Table 4).

In the optical colors (Figure 10), the nuclei of the two galaxies (cyan open diamond 3 and open black circle 7) are quite red, meaning high extinctions and/or age. Since they were undetected with GALEX, we are not able to tightly constrain their ages (see Table 4). In the case of the "bright spot" in the NGC 2856 disk, the optical colors can only constrain the age between 5 and 1500 Myr. The lack of detection in the NUV, however, further constrains the age to be $\gtrsim 400$ Myr (see Figure 11).

We also attempted to model the ages of the diffuse emission in the two galaxian disks and the northern tail by subtracting the clump light from the total emission. We modeled the starformation history of the diffuse emission in two ways: as an instantaneous burst and as continuous star formation. With the exception of ruling out extremely young ages (1-5 Myr), we cannot strongly constrain the age of the diffuse emission. For the instantaneous burst models, we get upper limits to the ages of the diffuse emission in the NGC 2854 and NGC 2856 disks of $1.5 \mathrm{Gyr}$ and $400 \mathrm{Myr}$, respectively. This implies that there are some recently formed stars in the disks outside the regions we defined as clumps. This does not, however, rule out an additional underlying older stellar population in the disks.

\subsection{Spitzer Colors}

Figure 12 shows the Spitzer [3.6 $\mu \mathrm{m}]-[4.5 \mu \mathrm{m}]$ versus [4.5 $\mu \mathrm{m}]-[5.8 \mu \mathrm{m}]$ colors for the clumps in the NGC 2856 tail (magenta diamonds), the NGC 2856 disk (cyan open diamonds), and the NGC 2854 disk (black open circles). A similar plot for [4.5]-[5.8] versus [5.8]-[8.0] is provided in the Appendix. These colors are compared to the colors of the Arp 107 and Arp 82 clumps (green diamonds, from Smith et al. 2005a and Hancock et al. 2007), Galactic interstellar dust (blue Xs; from Flagey et al. 2006), M0III stars (open blue square, from Cohen 2005, private communication), and field stars (magenta open triangle, from Whitney et al. 2004), as well as the total colors for the NGC 2856 tail region (red filled triangle).

As shown in Figure 12, [3.6]-[4.5] $\approx 0.0$ for both stars and Galactic dust. Global values for both interacting and spiral galaxy disks are also close to this value (Smith et al. 2007), as are the clumps in the disks of Arp 285, 107, and 82 (Figure 12). The [5.8]-[8.0] colors of most of the clumps in the three Arp systems are similar to those of interstellar matter and redder than stars, as expected since these bands are likely dominated by interstellar dust emission. The [4.5]-[5.8] colors of the Arp clumps are mainly between those of stars and interstellar matter (Figure 12), suggesting contributions from both. The very red [4.5]-[5.8] color of clump 3 in the Arp 285 tail compared to the clumps in Arp 107 and Arp 82 and the other clumps in Arp 285 (Figure 12) implies more contributions from interstellar matter. This is consistent with the very young age determined from the optical colors (Table 4).

To disentangle the contributions from starlight and dust to the Spitzer bands, the results of our stellar-population synthesis (Section 4.2) are helpful. In Figure 13, we plot the full optical-mid-infrared spectral-energy distribution (SED) for tail clump 3. We superimpose on this plot our best-fit Starburst99 model ( $4 \mathrm{Myr}, E(B-V)=0.1)$, along with models that span our $1 \sigma$ uncertainty ( $68 \%$ confidence) in the age (3-6 Myr). In addi- 


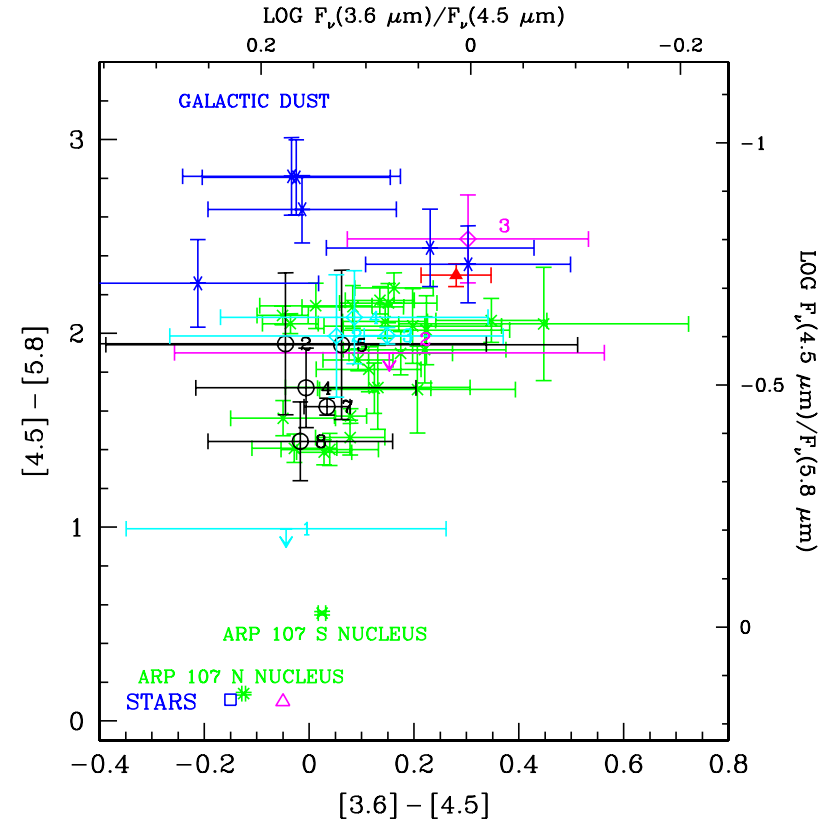

Figure 12. The Spitzer [4.5]-[5.8] vs. [3.6]-[4.5] color-color plot, showing the location of the clumps in the NGC 2856 tail (magenta open diamonds), the NGC 2856 disk (cyan open diamonds), and the NGC 2854 disk (black open circles). The clumps are labeled. The colors of M0III stars (open dark blue square), from M. Cohen (2005, private communication), and the mean colors of the field stars of Whitney et al. (2004) (magenta open triangle) are also shown. The colors of normal stars all lie within 0.5 mag of 0,0 in this plot $(\mathrm{M}$ Cohen 2005, private communication). We have also plotted the locations of the clumps in Arp 107 and Arp 82 as green asterisks (Smith et al. 2005b; Hancock et al. 2007), excluding likely foreground stars, background quasars, upper limits, and point with uncertainties $>0.5 \mathrm{mag}$. The observed Spitzer colors (Flagey et al. 2006) for diffuse dust toward several positions in the Milky Way are also plotted (blue Xs). The red diamond shows the colors of the $25^{\prime \prime} .6 \times 9$.' 7 region enclosing all four knots in the northern tail. The error bars include both statistical uncertainties and an uncertainty in the colors due to the varying sky annuli (see text).

tion, we include a theoretical dust spectrum from Draine \& Li (2007). This dust spectrum shows the broad polycyclic aromatic hydrocarbon (PAH) mid-infrared emission features, as well as a "hot dust" continuum. The plotted dust spectrum was calculated with the solar neighborhood interstellar radiation field, scaled up by a factor of $U=100$. It uses a PAH-to-total-dust mass ratio of $q_{\mathrm{PAH}}=4.6 \%$. The dust SED in our wavelength range does not vary much with $U$; lowering $q_{\mathrm{PAH}}$ weakens the PAH features (Draine \& Li 2007). The dust model has been scaled to fit the observed $8 \mu \mathrm{m}$ flux. The solid line in this plot is the combined stellar-dust spectrum. The H $\alpha$ contribution to the SDSS $r$ band is clearly visible in this plot. A contribution to the broadband $3.6 \mu \mathrm{m}$ Spitzer flux from the $3.3 \mu \mathrm{m}$ PAH feature is also apparent.

Figure 13 indicates that, in addition to the PAH contribution to the $3.6 \mu \mathrm{m}$ band and the modeled stellar component, the $3.6 \mu \mathrm{m}$ and $4.5 \mu \mathrm{m}$ fluxes of tail clump 3 also include another component. Either there is a significant "hot dust" contribution to these bands, as indicated by the Draine \& Li (2007) model, or there is a second underlying older stellar component that is not revealed by the optical/UV population synthesis.

For comparison to tail clump 3, in Figure 14 we plot the SED of clump 2 in the NGC 2856 disk. Although this also has a very young stellar population (see Table 4), it has a much more reddened SED than the tail clump because of much higher extinction. Also, although PAH emission is clearly present at

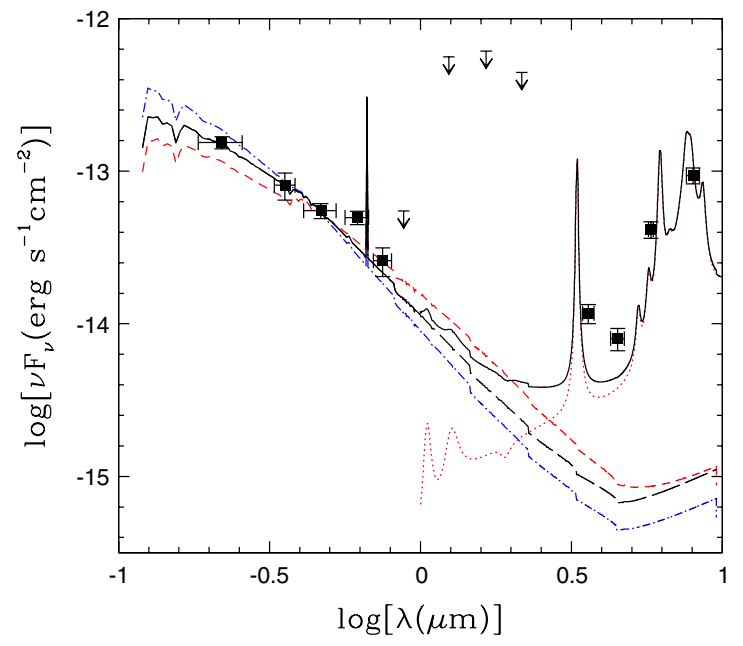

Figure 13. The UV-mid-IR SED of clump 3 in the northern tail (filled squares), including the upper limit in the SDSS $z$-band (arrow). The 2MASS upper limits are also shown. The long dashed black curve is the best-fit solar metallicity population synthesis model (4 Myr, $E(B-V)=0.1)$. The dot-dashed blue and short-dashed red curves show the youngest and oldest solar metallicity models respectively, with their associated best-fit extinctions. All of the models have been normalized to the $g$-band flux. The red dotted curve is the Draine \& Li (2007) Milky Way dust model with $U=100$ and $q_{\mathrm{PAH}}=4.6 \%$, scaled to the $8 \mu \mathrm{m}$ flux. The solid black curve is the sum of these three components. Note the contribution from the $3.3 \mu \mathrm{m}$ PAH feature to the $3.6 \mu \mathrm{m}$ Spitzer band, and the $\mathrm{H} \alpha$ contribution to the $r$ band. Also note that the $3.6 \mu \mathrm{m}$ and $4.5 \mu \mathrm{m}$ fluxes are much higher than expected from the stellar population synthesis model, suggesting contributions from either hot dust, as in the dust model shown, or a second colder stellar population undetected in the population synthesis.

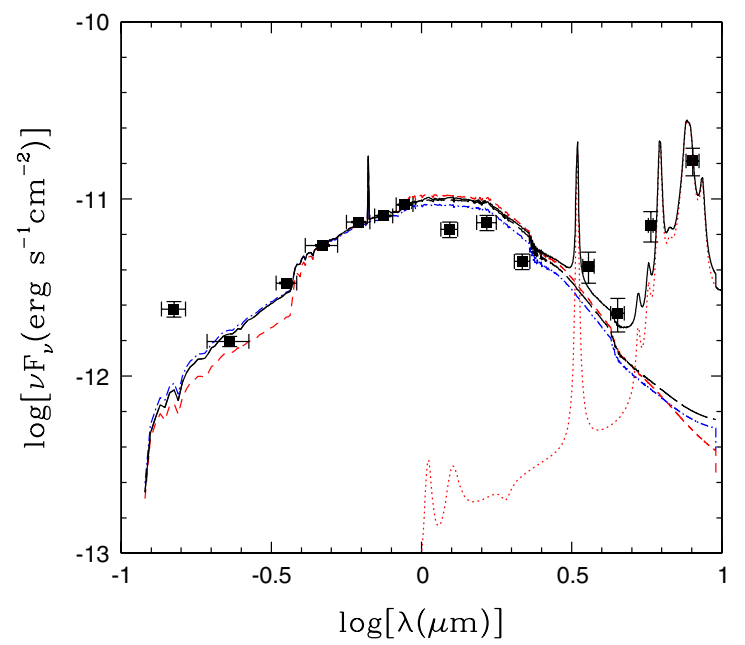

Figure 14. The UV-mid-IR SED of clump 2 in the NGC 2856 disk. Symbols and curves are as in Figure 13. Note that, although the age is similar to that of tail clump 3 (Table 4), the SED is very different because of the higher extinction. Starlight contributes a higher fraction of the 3.6 and $4.5 \mu \mathrm{m}$ fluxes in this clump than in tail clump 3.

$8 \mu \mathrm{m}$, in the 3.6 and $4.5 \mu \mathrm{m}$ bands most of the emission is stellar, in contrast to clump 3 in the tail.

The "bright spot" in the NGC 2856 disk (cyan clump 1) is undetected at $5.8 \mu \mathrm{m}$ and $8.0 \mu \mathrm{m}$, with a very blue [4.5][5.8] upper limit compared to the other clumps (Figure 12). This suggests that this region has an older stellar population than the other disk clumps. As noted earlier, with the available optical data we could not strongly constrain the age of this clump (Table 4), however, the lack of an NUV detection points to an 
Table 5

Optical Absolute Magnitude Ranges for Various Objects

\begin{tabular}{lcccc}
\hline \hline Class object & $M_{B}$ & $M_{V}$ & $M_{R}$ & Notes and references \\
\hline Arp 285 tail clumps & -12.0 to -10.6 & -12.3 to -10.8 & -12.3 to -10.9 & This work $^{\mathrm{a}}$ \\
NGC 2856 "bright spot" & -13.6 & -14.3 & -15.0 & This work $^{\mathrm{a}}$ \\
Other Arp 285 disk clumps & -15.9 to -12.1 & -17.1 to -13.2 & -17.8 to -13.3 & This work $^{\mathrm{a}}$ \\
Nearby dwarf irregular galaxies & -18 to $-8 ;$ median $=-13.2$ & & Karachentsev et al. (2004) \\
Local group irregular Leo A & -11.3 & & Karachentsev et al. (2004) \\
Local group irregular GR 8 & -12.0 & & Karachentsev et al. (2004) \\
M81 dwarf A & -12.4 & & Patterson \& Thuan (1996) \\
Tidal dwarf galaxies & -17 to -12.5 & & b \\
Arp 82 tail clumps & & -16 to -12 & -15.9 to -13.9 & Hancock et al. (2007) \\
Super-star clusters & & -11.3 & c \\
30 Dor R136 star cluster in LMC & & & O'Connell et al. (1994) \\
\hline
\end{tabular}

Notes.

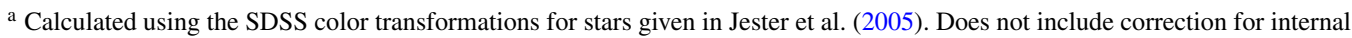
extinction.

b Weilbacher et al. (2003) and Higdon et al. (2006). Fainter end of NGC 5291 TDG range extrapolated from high end assuming constant $B-[3.6]$ color.

${ }^{c}$ Holtzman et al. (1992, 1996); O'Connell et al. (1994); Whitmore et al. (1993); Whitmore \& Schweizer (1995); Schweizer et al. (1996); Watson et al. (1996).

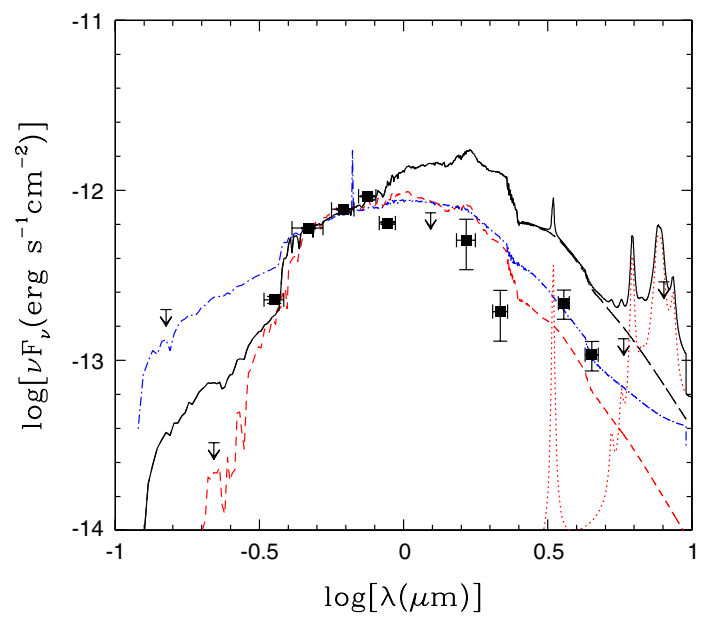

Figure 15. The UV-mid-IR SED of the "bright spot" at the edge of the NGC 2856 disk. Symbols and curves are as in Figure 13. Note the upper limits at $5.8 \mu \mathrm{m}$ and $8.0 \mu \mathrm{m}$, as well as the GALEX upper limits. Starlight can account for the $3.6 \mu \mathrm{m}$ and $4.5 \mu \mathrm{m}$ emission.

older age (see Section 4.2). The Spitzer results are consistent with this conclusion. This shows that Spitzer mid-infrared data may be useful for breaking the age-extinction degeneracy in optical colors. In Figure 15, we plot the SED for the "bright spot," with the best fit from the optical data shown. The NUV limit plotted shows the additional constraint on the age. The SED plot shows that the $3.6 \mu \mathrm{m}$ and $4.5 \mu \mathrm{m}$ emission is dominated by starlight, with very little if any dust contributing.

The two Arp 285 nuclei have [4.5]-[5.8] and [5.8]-[8.0] colors similar to the other clumps, implying nuclear starbursts. This is in contrast to the Arp 107 nuclei (Figure 12), which have older stellar populations (Smith et al. 2005a). The two nuclei in Arp 82, like those in Arp 285, have Spitzer colors of star-forming regions (Hancock et al. 2007).

\subsection{Absolute Magnitudes and Masses}

In Table 5, we compare the absolute optical magnitudes of the NGC 2856 tail clumps with dwarf galaxies, candidate tidal dwarf galaxies (TDGs), "super-star clusters" (SSCs), and the tail clumps in Arp 82. The Arp 285 tail clumps are lower luminosity than most nearby irregular galaxies and TDGs, and are near the lower end of the range for SSCs. The faintest Arp 285 tail clump, clump 4, is somewhat less luminous than R136, the bright star cluster in 30 Doradus in the Large Magellanic Cloud (O'Connell et al. 1994). In contrast, the "bright spot" in the NGC 2856 disk is near the median for dwarf irregular galaxies.

For the Arp 285 clumps, in Table 6 we give the range of stellar masses inferred from the Starburst99 models. In this table, we also provide stellar masses of various other objects for comparison. The tail clumps are similar in mass to Galactic globular clusters, but have lower stellar masses than those inferred for TDGs and dwarf irregular galaxies. The mass of the NGC 2856 disk "bright spot" is near the median for dwarf irregular galaxies.

The $3.6 \mu \mathrm{m}$ Spitzer band is sometimes used as a tracer of stellar mass (e.g., Li et al. 2007). However, our SED plots (Figures 13-15) show that the stellar mass-to-3.6 $\mu \mathrm{m}$ luminosity varies significantly from clump to clump, depending upon the star-formation rate and gas-to-star ratio. This is illustrated in Figure 16, where we plot the stellar mass of the clump determined from the population synthesis model against the $3.6 \mu \mathrm{m}$ luminosity. We have also included values for the clumps in Arp 82 (Hancock et al. 2007). On this curve, we have superimposed lines of constant stellar mass-to-light ratios of $M / L_{3.6}=1 M_{\odot} / L_{\odot}($ solid line $)$ and $M / L_{3.6}=10 M_{\odot} / L_{\odot}$ (dotted line), where $L_{\odot}$ is the bolometric luminosity of the Sun. This plot shows that the NGC 2856 tail clumps and four clumps in NGC 2854 (clumps 1, 3, 5, and 6, in the outer parts of the spiral arms) have lower $M / L_{3.6}$ ratios than the other clumps, which are close to the $M / L_{3.6}=10 M_{\odot} / L_{\odot}$ line. This indicates that contributions from hot dust and/or the $3.3 \mu \mathrm{m}$ PAH feature to the $3.6 \mu \mathrm{m}$ flux are significant in the tail and outer spiral arm regions. Thus caution should be used in utilizing the Spitzer $3.6 \mu \mathrm{m}$ band to estimate stellar masses in star-forming regions. For example, for clump 3 in the tail, the stellar mass is $\sim 1 / 30$ th that expected based on the $M / L_{3.6}=10 M_{\odot} / L_{\odot}$ relationship. 
Table 6

Stellar Mass Ranges for Various Objects

\begin{tabular}{lcc}
\hline \hline & Mass & Notes and references \\
\hline Arp 285 Tail clumps & $2 \times 10^{4}-8 \times 10^{5} M_{\odot}$ & This work $^{\mathrm{a}}$ \\
NGC 2856 "bright spot" & $\sim 10^{8} M_{\odot}$ & This $^{\mathrm{w}}$ wrk $^{\mathrm{a}}$ \\
Other non-nuclear Arp 285 disk clumps & $3 \times 10^{6}-10^{9} M_{\odot}$ & This work $^{\mathrm{a}}$ \\
Arp 285 Nuclei & $5 \times 10^{9} M_{\odot}$ & This work $^{\mathrm{a}}$ \\
Dwarf irregular galaxies & $6.3 \times 10^{6}-1.2 \times 10^{9} M_{\odot} ;$ Median $=10^{8} M_{\odot}$ & ${\text { Hunter \& Gallagher }(1985)^{\mathrm{b}}}_{\text {Tidal dwarf galaxies }}$ \\
Arp 82 tail clumps & $2 \times 10^{6}-5 \times 10^{8} M_{\odot}$ & $\mathrm{c}$ \\
Local group globular clusters & $5 \times 10^{6}-8 \times 10^{7} M_{\odot}$ & $\mathrm{d}$ \\
& $3 \times 10^{3}-3 \times 10^{6} M_{\odot} ;$ Median $=1.3 \times 10^{5} M_{\odot}$ McLaughlin \& van der Marel (2005)
\end{tabular}

Notes.

a The uncertainties on these masses are a factor of 2-5.

b Converted to $H_{0}=75 \mathrm{~km} \mathrm{~s}^{-1} \mathrm{Mpc}^{-1}$.

${ }^{c}$ Braine et al. (2001) and Higdon et al. (2006).

${ }^{\mathrm{d}}$ Hancock et al. (2007). Uncertainties are a factor of $\sim 2-10$.

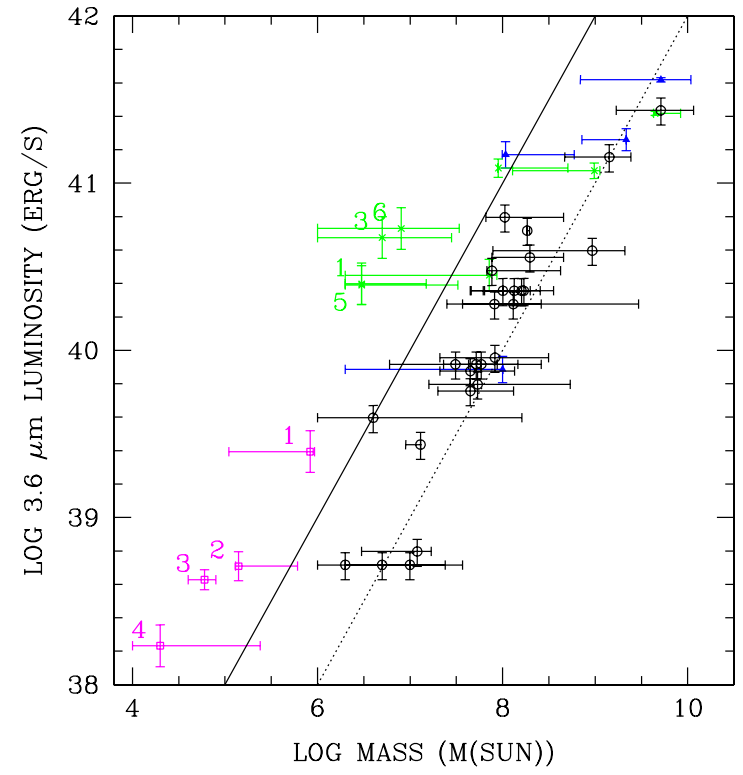

Figure 16. The $3.6 \mu \mathrm{m}$ luminosity of the Arp 285 clumps, compared to their stellar masses implied by the population synthesis models. The magenta open squares are the tail clumps, the blue filled triangles are clumps in the NGC 2856 disk, and the green crosses are the NGC 2854 disk clumps. The black open circles are the Arp 82 clumps, from Hancock et al. (2007). A constant $M / L_{3.6}$ ratio of $1 M_{\odot} / L_{\odot}$ is represented by the solid black line, while the dotted line is $M / L_{3.6}=$ $10 M_{\odot} / L_{\odot}$. The $3.6 \mu \mathrm{m}$ luminosity was calculated assuming the FWHM of the bandpass $\Delta v$ of $16.23 \times 10^{12} \mathrm{~Hz}$.

\section{A NUMERICAL MODEL OF THE ENCOUNTER}

To interpret these observational results in terms of the dynamical and star-forming history of Arp 285, we have constructed a numerical simulation of the Arp 285 interaction using the smoothed particle hydrodynamics (SPH) code of Struck (1997). This code was previously used to model Arp 284 (Struck \& Smith 2003), IC 2163/NGC 2207 (Struck et al. 2005), Arp 107 (Smith et al. 2005a), and Arp 82 (Hancock et al. 2007).

\subsection{Constraints on the Model}

Arp 285 is less symmetric than the ring galaxies or planar fly-by encounters such as M51, NGC 2207/IC2163, and Arp 82. The collisional morphology of Arp 285 appears somewhat similar to that of Arp 284, an asymmetric ring/tail galaxy (NGC 7714) with an edge-on companion (NGC 7715). The sub- stantial bridge and tail of NGC 2854, like those of NGC 7715, lead us to believe that it suffered a strong prograde encounter. There are also similarities between NGC 7714 and NGC 2856. The optical images show that the "bright spot" in the northwestern section of the NGC 2856 disk (disk clump 1) is part of an arc-like structure (see Figures 1 and 2). This arc is reminiscent of the partial ring in NGC 7714 (see the Arp (1966) photograph of Arp 284), which has been successfully modeled by an offcenter collision (Struck \& Smith 2003). It is also reminiscent of the "ripples" in Arp 227, which were also modeled by a ring galaxy-like collision by Wallin \& Struck-Marcell (1988).

There are some differences between NGC 2856 and NGC 7714, however. In contrast to NGC 7714, NGC 2856 lacks strong tidal tails, except for the northern tail perpendicular to the disk and a short $\mathrm{HI}$ extension to the northwest (Chengalur et al. 1994). This suggests that NGC 2856 did not experience the encounter as very prograde. It also does not have the fan-like form common to strong retrograde encounters. This suggests that the orbital path of the two galaxies is at a large angle to the plane of the NGC 2856 disk.

These considerations give us some idea of the type of collision that produced the current morphologies. In our simulation of this encounter, we have limited ourselves to the goal of reproducing the large-scale morphological structures, but have not attempted to simulate internal disk structures nor match the system kinematics in any detail.

One key feature we would like the models to help us understand is the beads in the tail north of NGC 2856. We have considered several conceptual ideas for the origin of this material. The H I morphology suggests that this material is an extension of the bridge from NGC 2854, though the optical observations look as though the bridge curves away from that direction before connecting to the bead region. It may be that the bridge is in fact a tidal tail, which is merely projected onto NGC 2856, not connected. However, the H I kinematics indicate that this is unlikely. Moreover, the bead material seems strongly affected by the gravitational potential of NGC 2856.

Thus, it seems likely that the bead material is accreting onto the halo of NGC 2856 from the bridge. There are two possibilities for how this occurs: (i) as infall through the disk of NGC 2856 and out the other side, or (ii) by swinging around that disk to the other side. It is difficult to distinguish between these two scenarios observationally. In option (i) we can imagine that clouds pushing through the NGC 2856 disk are shocked and compressed. This may trigger star cluster formation, accounting for the beads. We would naively expect this process to be 

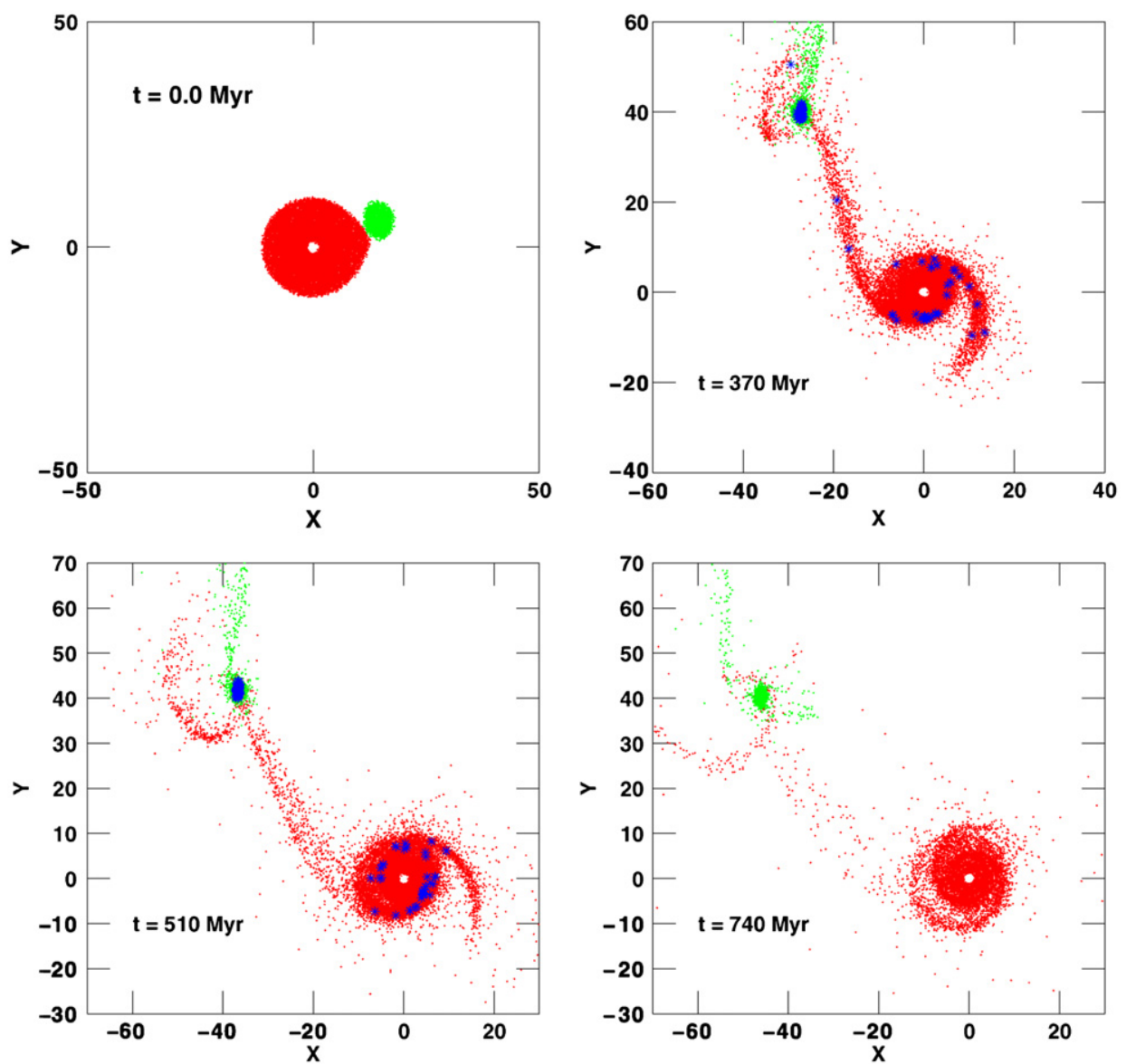

Figure 17. Snapshots of the model gas disks. The stellar appearance is similar. Red particles originated in the primary galaxy, green in the companion. The top-left panel shows a time near closest approach $(T=0 \mathrm{Myr})$. The companion has swung in from the lower left, and swings around to an apogalacticon point at later times. The upper-right panel and the lower left are at times near the present $(T=370 \mathrm{Myr}$ and $510 \mathrm{Myr}$, respectively). The lower-right panel is at a later time $(T=$ $740 \mathrm{Myr}$ ), when the companion begins to fall back to merge with the primary. In the first three panels every third gas particle is plotted with a dot. In the second and third panels blue asterisks mark star-forming particles, except those within $5 \mathrm{kpc}$ of the primary center, which were omitted for clarity. The star-forming region in the northern tail was produced from gas accreted from the companion, while the star-forming regions in the central region of the northern galaxy were produced from gas that originated in the northern galaxy. The star-forming regions in the bridge, southern galaxy, and southern tail were formed from gas that originated in the southern galaxy. In the final panel only every fifth particle was plotted, to show the persistent spiral in the primary disk. The motion of the companion around the point of greatest separation is very slow, so little positional change is evident in the last three panels. Later time steps are shown in the Appendix.

sequential, so that the beads furthest from the disk are oldest. In contrast, in option (ii), a group of inflowing clouds pile up in the halo of NGC 2856 and collide with the material that arrived earlier. This could trigger star formation simultaneously at several locations. Thus option (i) would predict an age gradient, while for option (ii) we would expect roughly coeval clumps. With the available data, we cannot distinguish between these two possibilities, since the expected age gradient for option (i) is too small to measure. Assuming a nominal velocity for the tidal material away from the disk of $\sim 300 \mathrm{~km} \mathrm{~s}^{-1}$ and motion in the plane of the sky, for scenario (i) we would expect an age difference of $\sim 12$ Myr between the first and fourth clumps in the tail, and $\sim 4 \mathrm{Myr}$ between clumps 2 and 3. This is smaller than the uncertainties on the ages of these clumps (Table 4).

Another way to distinguish between these two scenarios is with numerical models of the interaction. For option (i), we were not able to construct a viable simulation with a small number of trial runs. The fundamental difficulty is that in order to produce the spirals and other tidal structures in NGC 2854 the collision must have a substantial prograde fly-by component with respect to NGC 2854. In that case, however, material accreted onto
NGC 2856 from NGC 2854 generally has too much relative angular momentum to fall directly onto the NGC 2856 disk. Because of this, we suspect that such models occupy a small volume of the collision parameter space. We have therefore chosen to focus on models for option (ii). These are discussed in the next two sections.

\subsection{Model Details}

In the SPH code, hydrodynamical forces are calculated on a grid with fixed spacing. Gravitational forces are computed between particles in adjacent cells, to capture local gravitational instabilities. The model galaxies have disks containing both gas particles and collisionless star particles, as well as rigid dark halo potentials (see Struck 1997 for details). Gas particles with densities exceeding a constant density threshold are identified as star-forming particles. These generally exceed the local Jeans critical mass. A number of simulations were run; we will only present the results of the best model.

The evolution of our numerical model for the Arp 285 system is presented in Figure 17, with additional time steps provided in the Appendix. We adopt the convention that the model primary corresponds to the southern galaxy NGC 2854 
and the companion to NGC 2856. The particles in Figure 17 are color coded according to their galaxy of origin, with red particles originating from the primary disk and green from the companion. A total of 13,590 star and 42,900 gas particles were used in the primary disk and 5640 star and 5640 gas particles in the secondary disk. In this model, the length unit $=1.0 \mathrm{kpc}$, and the time unit is 200 Myr. Figure 17 shows four time steps in the simulation. The first plot (top left) shows the appearance in the plane of the sky near the time of closest approach, where the separation between the two galaxies is $\sim 12 \mathrm{kpc}$. The second plot (top right) shows the system $370 \mathrm{Myr}$ after closest approach, while the third (bottom left) shows its appearance $510 \mathrm{Myr}$ after closest approach. These two plots match approximately the observed appearance at the present time. The last plot shows the appearance $740 \mathrm{Myr}$ after closest approach, the predicted appearance in the future.

The radii of the primary star and gas disks are 6.0 and $10.8 \mathrm{kpc}$, respectively. The companion star and gas disk radii are both $3.6 \mathrm{kpc}$. The primary disk was set up in the $x-y$ plane. The companion disk is first set up in the $x-y$ plane, then rotated $40^{\circ}$ around the $y$-axis through its center, and then $90^{\circ}$ around the $z$-axis passing through its center. The relative orbit of the companion is in the $x-y$ plane, so from the point of view of the companion disk, the primary approaches at a fairly steep angle. In the companion disk of the model in Figure 17, the south side is the near side.

The orbit is counter-clockwise, as is the rotation of the primary, so it sees the encounter as very prograde. The companion disk rotation, in the $x-y$ plane before the tilts are applied, is clockwise. The initial $(x, y, z)$ position of the companion relative to the primary center is $(-8.9,-20.0,0.0) \mathrm{kpc}$. Its initial relative velocity is $(250,75,0) \mathrm{km} \mathrm{s}^{-1}$.

The form of the halo potential of the two galaxies is such that the acceleration of a test particle in this halo is

$$
a=\frac{G M_{h}}{\epsilon^{2}} \frac{r / \epsilon}{\left(1+r^{2} / \epsilon^{2}\right)^{n_{h}}},
$$

where $M_{h}$ is a halo mass scale, $\epsilon$ is a core radius (set to 2.0 and $4.0 \mathrm{kpc}$ for the primary and companion, respectively), and the index $n_{h}$ specifies the compactness of the halo. For the primary we use $M_{h}=1.3 \times 10^{10} M_{\odot}$ and $n_{h}=1.2$, which gives a slightly declining rotation curve at large radii. For the companion we take $M_{h}=2.8 \times 10^{10} M_{\odot}$ and $n_{h}=1.35$, which gives a more rapidly declining rotation curve. The model includes the effects of dynamical friction with a Chandrasekhar-like frictional term (see Struck \& Smith 2003). The effects of this term are small except near the closest approach.

With these potentials, the halo masses for the primary and companion out to a radius of $12 \mathrm{kpc}$ (about the separation at closest approach) are $3.7 \times 10^{10} M_{\odot}$ and $3.4 \times 10^{10} M_{\odot}$, respectively, with a ratio of about 0.92 . This is in accord with the near equality of the $r$ and $i$ band luminosities of the two galaxies (Table 1).

\subsection{Model Results}

The general morphology of the system is quite well reproduced by the model, including the moderate countertail on NGC 2854 and the bridge (see Figure 17). A very close encounter is required to produce a bridge as massive as observed. On the other hand, the moderate-sized tail of the primary galaxy is the result of a prograde perturbation that was not prolonged. These facts, and the relatively large separation between the galaxies, argue that the relative orbit of the companion is quite elliptical, as in the model.

The model primary disk is more circular in appearance than that of NGC 2854. There are several possible reasons for the difference. The first is simply that the model disk should have a greater tilt relative to the plane of observation (here the $x-y$ plane). The primary disk in the model is in the $x-y$ plane of the sky. However, as noted in Section 3.2, based on extinction arguments and the $\mathrm{H}$ I velocity field, the real disk is somewhat inclined to the line of sight, with the south side closest. It is also possible that tidal stretching is responsible for the shape of the primary disk. However, in that case we might expect a longer and more massive tidal tail. This is a rather soft argument at present, but it does appear that the bar and the spiral arms of the primary disk are disproportionately strong relative to the tail. This suggests that the bar and spiral arms were present in the NGC 2854 disk before the encounter. This possibility was not included in the modeling.

In addition to the bridge, the model companion galaxy has two tidal tails, one made of material originating from the companion galaxy itself, and the other from material accreted from the primary galaxy along the bridge (see Figure 17). The tidal plume drawn off the companion disk is visible as the green feature extending northward in the last three panels of Figure 17. We equate this structure with the $\mathrm{H}$ I emission extending to the northwest in the Chengalur et al. (1994) $\mathrm{H} \mathrm{I}_{\mathrm{I}}$ maps, though it is not at the same position angle as in the observations, being oriented about $45^{\circ}$ too much to the north compared to the data. The red feature extending to the northeast in Figure 17 we associate with the northern $\mathrm{H}$ I tail containing the "beads" of star formation. As with the other feature, the position angle of the model tail is somewhat off from the observed orientation.

In the model, the disk of the companion was tilted relative to the direction of the encounter, so the perturbation had both an orthogonal, ring-galaxy-like component, and a retrograde component. Waves with circular arc-like components develop in the disk of the companion. This behavior might account for the northwest arc-like structure in NGC 2856 containing the "bright spot."

As with NGC 2854, the observed structure of NGC 2856 also shows a bar and internal arms. However, in this galaxy the structure of the bar is rather irregular. The simulation shows that a large mass of gas loses angular momentum as a result of the encounter, and forms a compressed inner disk or bar. Thus, the bar in NGC 2856 may be the result of the collision, and may not have existed before the encounter.

The evolution of the bridge in this model is especially interesting. Because of its elliptical trajectory, the companion speeds past its point of closest approach as the bridge begins to form. As the bridge initially stretches outward from the primary center, it lags behind the companion. Later, the companion nears its apogalacticon relative to the primary, and slows, so that the bridge catches up to it. The bridge material has a significant angular momentum relative to the companion center, so that the outermost points swing around to the far side of the companion. Shortly thereafter the bridge material begins to pile up at an outer radius northeast of the companion. As time goes on, more bridge material streams into this pile-up region, and compression drives star formation. In Figure 17, star-forming gas particles are marked with cyan asterisks. In the second panel, one asterisk is visible in the pile-up region. Comparison of different time steps and different models shows 


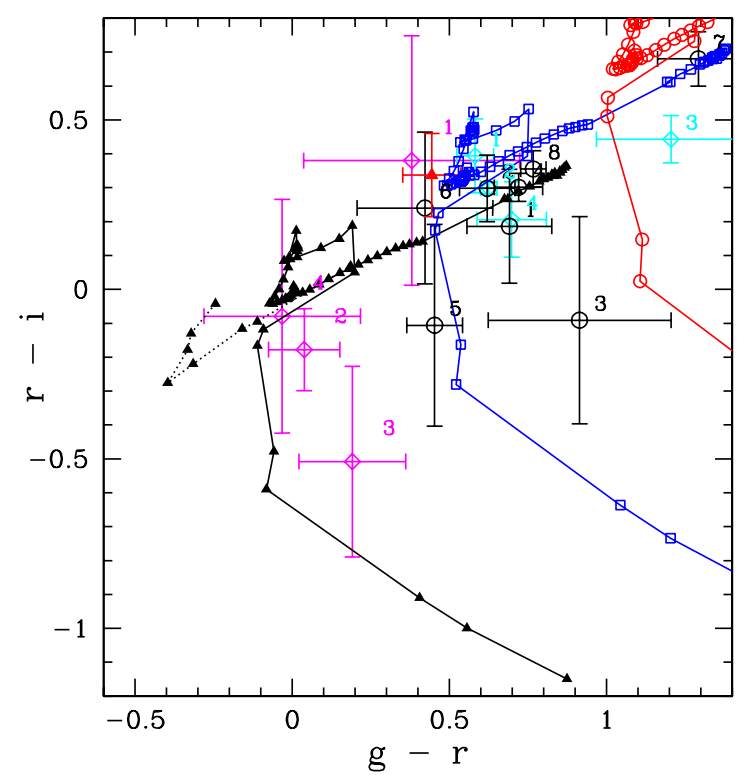

Figure A1. The $r-i$ vs. $g-r$ colors of the clumps in the NGC 2856 tail (magenta open diamonds), the NGC 2856 disk (cyan open diamonds), and the NGC 2854 disk (black open circles), compared with solar metallicity Kroupa IMF instantaneous burst population synthesis models with extinction of $E(B-$ $V)=0$ (black filled triangles), 0.5 (blue open squares), and 1.0 (red open circles). The clumps are identified by their numbers in Table 2, labeled in the same color. For the zero extinction model two curves are shown: with (solid line) and without (dotted line) $\mathrm{H} \alpha$. The model ages start with an age of $1 \mathrm{Myr}$ for the point at the lower-left end of the curve, increased by $1 \mathrm{Myr}$ steps to $20 \mathrm{Myr}$, then by $5 \mathrm{Myr}$ steps to $50 \mathrm{Myr}$, then $10 \mathrm{Myr}$ steps to $100 \mathrm{Myr}, 100 \mathrm{Myr}$ steps to $1 \mathrm{Gyr}$, and $500 \mathrm{Myr}$ steps to $10 \mathrm{Gyr}$. The red filled diamond shows the colors of the $25^{\prime \prime} 6 \times 9$ '.7 region enclosing all four knots in the northern tail.

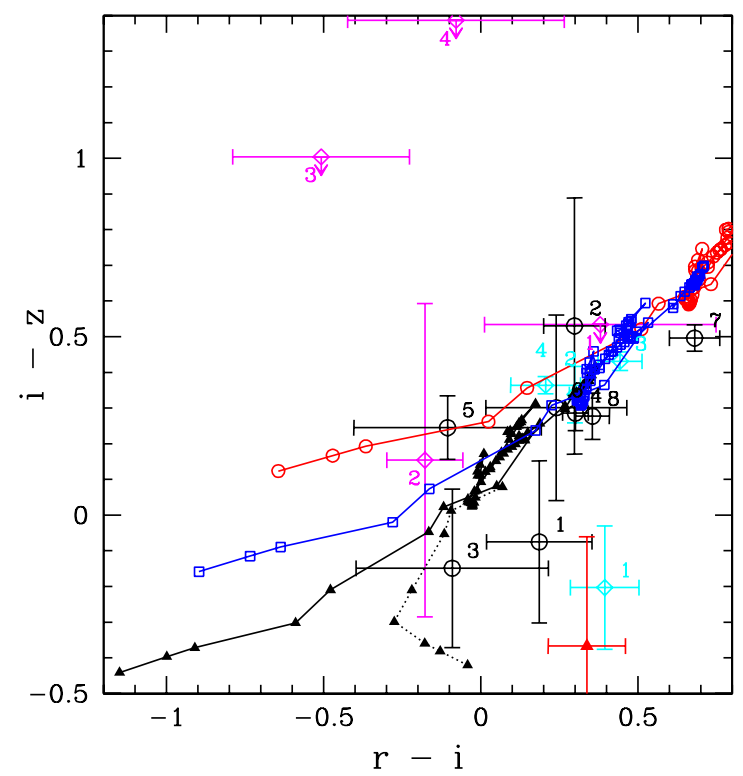

Figure A2. The $i-z$ vs. $r-i$ colors of the clumps in the NGC 2856 tail (magenta open diamonds), the NGC 2856 disk (cyan open diamonds), and the NGC 2854 disk (black open circles). The clumps are identified by their numbers in Table 2, labeled in the same color. Superimposed on this plot are solar metallicity Kroupa IMF instantaneous burst population synthesis models with extinction of $E(B-V)=0$ (black filled triangles), 0.5 (blue open squares), and 1.0 (red open circles). The model ages start with an age of $1 \mathrm{Myr}$ for the point at the lower-left end of the curve, increased by $1 \mathrm{Myr}$ steps to $20 \mathrm{Myr}$, then by 5 Myr steps to $50 \mathrm{Myr}$, then $10 \mathrm{Myr}$ steps to $100 \mathrm{Myr}, 100 \mathrm{Myr}$ steps to $1 \mathrm{Gyr}$, and $500 \mathrm{Myr}$ steps to $10 \mathrm{Gyr}$. The red filled triangle shows the colors of the $255^{\prime \prime} 6 \times$ 9!'7 region enclosing all four knots in the northern tail. For the zero extinction model two curves are shown: with (solid line) and without (dotted line) $\mathrm{H} \alpha$.

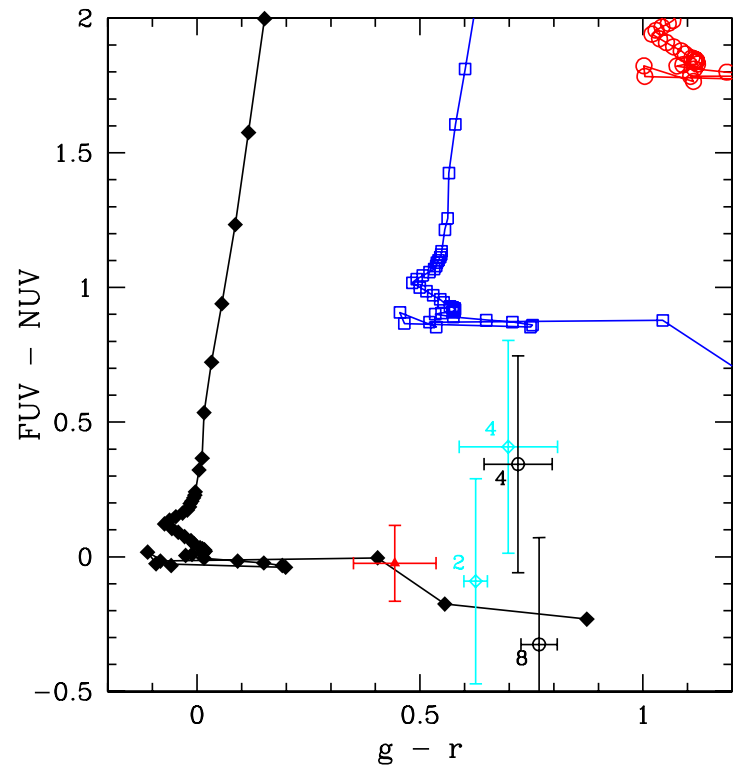

Figure A3. The FUV-NUV color of the 25".6 × 9".7 region in the northern tail, plotted against $g-r$ (red filled triangle). The clumps in the NGC 2856 disk (cyan open diamonds) and the NGC 2854 disk (black open circles) are also plotted. Solar metallicity Kroupa IMF instantaneous burst population synthesis model colors are also shown, with extinction of $E(B-V)=0$ (black diamonds), 0.5 (blue open squares), and 1.0 (red open circles). The model ages start with an age of $1 \mathrm{Myr}$ for the point at the lower-right end of the curve, increased by $1 \mathrm{Myr}$ steps to $20 \mathrm{Myr}$, then by $5 \mathrm{Myr}$ steps to $50 \mathrm{Myr}$, then $10 \mathrm{Myr}$ steps to $100 \mathrm{Myr}, 100 \mathrm{Myr}$ steps to $1 \mathrm{Gyr}$, and $500 \mathrm{Myr}$ steps to $10 \mathrm{Gyr}$. All models include $\mathrm{H} \alpha$.

that the star formation there is quite stochastic. Sometimes there are a number of star-forming particles there, and occasionally they line up like the observed "beads." Since the model does not accurately represent the effects of self-gravity across this pile-up region, the real environment may trigger more such star formation than in the model.

Material from both the accretion tail and the companion's plume eventually accrete onto the companion. Gas in the companion is compressed by the tidal perturbation, and experiences prolonged accretion. In the model, the density threshold for star formation is easily exceeded, and central star formation continues for some time. This is consistent with the uncertainties on the age of the stellar population in disk clump 3 (the nucleus) of NGC 2856 (Table 4).

Our simulation somewhat resembles models of polar ring formation via accretion from a companion (Reshetnikov et al. 2006), however, the two model galaxies merge before this polar ring proceeds very far in its development (see later time steps in the Appendix). The star clusters formed in the pile-up region will eventually be carried with the companion halo into the merger with the primary. They are likely to end up orbiting in the inner halo of the merger remnant and possibly adding to the globular cluster population there. This is in contrast to dwarf galaxies formed at the end of tidal tails, which may spend long periods in the outer halo.

\section{DISCUSSION}

The clumps in the northern tail of NGC 2856 are a striking example of the "beads on a string" phenomenon, in which star-forming regions are regularly spaced $\sim 1 \mathrm{kpc}$ apart along spiral arms and in tidal features (Elmegreen \& Efremov 1996). 


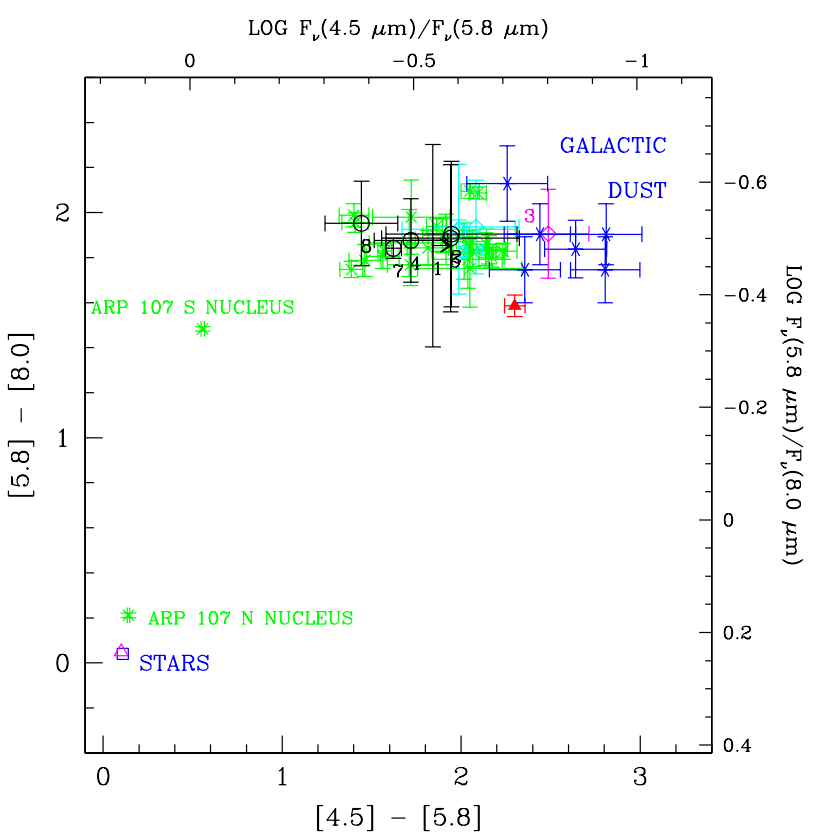

Figure A4. A Spitzer [5.8]-[8.0] vs. [4.5]-[5.8] color-color plot, showing the location of the NGC 2856 tail clumps (magenta open diamonds), the NGC 2856 disk clumps (cyan open diamonds), and the NGC 2854 clumps (black open circles), as well as the rectangular region in the tail including all four tail clumps (red filled triangle). The colors of M0III stars (open dark blue square), from M. Cohen (2005, private communication), and the mean colors of the field stars of Whitney et al. (2004) (magenta open triangle) are also shown. The colors of normal stars all lie within $0.5 \mathrm{mag}$ of 0,0 in this plot (M. Cohen 2005, private communication). The clumps associated with Arp 107 (Smith et al. 2005a) and 82 (Hancock et al. 2007) are also shown (green asterisks), except for excluding likely foreground stars, background quasars, upper limits, and point with uncertainties $>0.5 \mathrm{mag}$. Diffuse dust in the Milky Way is also plotted (blue Xs, from Flagey et al. 2006). The red diamond shows the colors of the $25^{\prime \prime} .6 \times$ 9'.7 region enclosing all four knots in the northern tail. The error bars include both statistical uncertainties and an uncertainty in the colors due to the varying sky annuli (see text).
Such a "beads on a string" morphology may be indicative of the gravitational collapse of interstellar gas clouds under selfgravity (Elmegreen \& Efremov 1996). Similar "bead strings" are also seen in the interacting systems IC 2163/NGC 2207 (Elmegreen et al. 2006) and Arp 82 (Hancock et al. 2007). In IC 2163/NGC 2207, the "beads" resolve into associations of star clusters in higher-resolution Hubble Space Telescope (HST) images (Elmegreen et al. 2006). In general, HST images of nearby galaxies show that young star clusters themselves tend to be clustered into complexes with typical sizes of $\sim 1$ kpc (Zhang et al. 2001; Larsen 2004; Bastian et al. 2005). As noted earlier, clumps 3 and 4 in the NGC 2856 tail have multiple peaks visible in the SDSS images. It is possible that the other two tail clumps, clumps 1 and 2, which are unresolved in the SDSS images, will also resolve into multiple star clusters at higher resolution.

The optical-UV colors of the clumps in the NGC 2856 tail are very blue, and imply ages of only $\sim 4-20$ Myr. This is much younger than the time since the point of closest approach between the two galaxies, showing that there is a time delay between the initiation of star formation and the time of closest approach between the two galaxies. According to our numerical model of this system, there should be an underlying older stellar component in this tail, made of stars stripped from the NGC 2854 disk. Diffuse optical light is clearly present between the clumps, and the stellar tail extends $41^{\prime \prime}(7.8 \mathrm{kpc})$ to the north beyond clump 4 . However, we are not able to tightly constrain the age of this diffuse stellar population. Thus it is unclear from the available data whether a stellar component to the tail existed before the current star-forming episode.

The tail clumps are of lower mass than the concentrations in other tails previously classified as TDGs (see Table 6). They are more similar in mass to globular clusters than dwarf irregular galaxies. Because of their low mass and the lack of $24 \mu \mathrm{m}$ detections and calibrated $\mathrm{H} \alpha$ measurements, it is not possible to get accurate star-formation rates for these clumps. Very roughly, using the $8 \mu \mathrm{m}$ luminosity for clump 3 in the northern tail, and
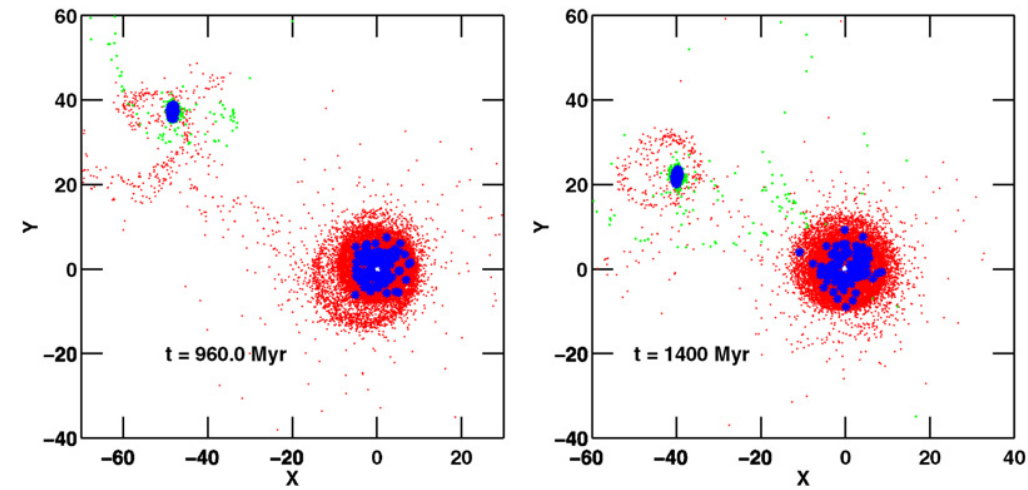

Figure A5. Snapshots of the model gas disks, as in Figure 17 in the main paper, but at two late times. Red particles originated in the primary galaxy, green in the companion. Blue asterisks mark star-forming particles. The point of greatest separation is reached at a time between the last panel of Figure 17 and the left panel of this figure. In the latter, the two galaxies are just beginning to fall back together. By the time of the right panel, they have moved noticeably closer, and developed a significant infall velocity. The comparison of this figure to Figure 17 reveals several interesting features. First, the companion disk settles into a compact, bar-like configuration, which does not change greatly with time. With a relatively high gas density and ongoing accretion, it maintains a moderate level of star formation continuously, for more than 1 Gyr. This feature alone has interesting ramifications for other systems, e.g., by contrast with the post-starburst companion of M51. Star formation also continues in the primary (NGC 2854), but it is more concentrated in the central regions. As the spiral waves damp away, star formation also vanishes in the outer disk. Similarly the tidal features around the companion become ever more diffuse, and are generally unable to exceed the density threshold for star formation. The time period when they could do so was generally a short one around the time of the middle panels of Figure 17. Qualitatively these results agree with the observationally determined ages of Table 4 . The tidal material that is not captured or dispersed tends to form an off-center, polar-like ring. (Since it has not settled into a fundamental plane, it is not truly a polar ring.) This ring probably has a lifetime of about 1 Gyr. Given the initial close encounter and the subsequent effects of dynamical friction, we expect the companion to impact the primary disk at the next close approach. Since the gas ring has a low surface density, it will not survive the impact. Encounters with slightly different collision parameters might not impact on the second, in which case the polar ring could survive for a somewhat longer time. We are not suggesting that this type of collision is a means of forming long-lived polar ring galaxies, but it is a curiosity. 
assuming the $8 \mu \mathrm{m}-24 \mu \mathrm{m}$ relationship found for M51 clumps of Calzetti et al. (2005) and their correlation between starformation rate and $24 \mu \mathrm{m}$ luminosity, we find a star-formation rate for this clump of $\sim 10^{-3} M_{\odot} \mathrm{yr}^{-1}$. This value is very uncertain due to the low mass and the bootstrapping from the $8 \mu \mathrm{m}$ flux.

In our model, gas from the bridge falling into the potential of the companion overshoots the companion, piling up in an accretion tail on the far side of the companion. Star formation occurs in this region. Our model suggests that the "beads on the string" may be the result of stochastic processes, albeit in a density enhanced pileup zone. It is possible that local selfgravity is pulling clumps together. The spacing between the star-forming regions in the model is comparable to the scale of local self-gravity in the code. At most time steps, the star formation is found in a couple of isolated clumps, without any "bead" appearance. Thus it appears we see this feature at a favorable time.

The Arp 285 tail is not unique. Accretion from a companion along a bridge may have produced the star-forming "countertails" in Arp 105 (Duc \& Mirabel 1994; Duc et al. 1997) and Arp 104 (Roche 2007). In addition, the inner tail on the western side of NGC 7714, which also has strong star formation (Smith et al. 1997), may have formed from accretion from the companion (Struck \& Smith 2003).

Our model suggests that the so-called "bright spot" in the northwestern portion of the NGC 2856 disk, and its associated arc, were likely to be caused by a ring-like perturbation of the disk by an encounter which was mainly perpendicular to the plane of the NGC 2856 disk. The age of the stellar population in this region is estimated to be between 400 and $1500 \mathrm{Myr}$, while the interaction model indicates that the point of closest approach between the galaxies occurred between about 300 and $500 \mathrm{Myr}$ ago. This is consistent with the idea that the brightness of this "spot" may be due to the past star formation triggered by the encounter.

\section{SUMMARY}

We have investigated star formation in the interacting galaxy pair Arp 285 using Spitzer infrared, GALEX ultraviolet, and ground-based optical data, and have constructed a numerical model of the interaction. The northern galaxy in this pair contains an unusual tail-like feature extending perpendicular to the disk. Our model suggests that this structure was created by gas from the companion falling into the gravitational potential of the disk and overshooting the disk.

A series of regularly-spaced knots of recent star formation are seen in this tail. Stellar population synthesis suggests that these knots have ages of $\sim 4-20 \mathrm{Myr}$ and masses in the range of globular clusters. The Spitzer 3.6 and $4.5 \mu \mathrm{m}$ fluxes from these tail clumps are higher than expected from the population synthesis, indicating that either a second older stellar population is present, or there are significant contributions to these bands from hot dust.

The "bright spot" in the NGC 2856 disk has an intermediateage stellar population (400-1500 Myr). This feature and its associated arc may have been caused by a ring-like disturbance from an encounter almost perpendicular to the plane of the disk. Its brightness might be due to past star formation triggered by the interaction.

We thank the Spitzer, GALEX, and SDSS teams for making this research possible. This research was supported by NASA
Table A1

Model Ages and Extinctions for Clumps with 0.2 Solar Metallicity Models ${ }^{\mathrm{a}}$

\begin{tabular}{|c|c|c|c|}
\hline Clump & $\begin{array}{c}\text { Age } \\
(\mathrm{Myr})\end{array}$ & $\begin{array}{c}E(B-V) \\
(\mathrm{mag})\end{array}$ & Colors used \\
\hline \multicolumn{4}{|c|}{ NGC 2856 tail clumps } \\
\hline 1 & $247 \pm{ }_{244}^{689}$ & $0.16 \pm{ }_{0.16}^{0.82}$ & $\mathrm{NUV}-g, g-r, r-i$ \\
\hline 2 & $5 \pm{ }_{1}^{20}$ & $0.3 \pm \pm_{0.3}^{0.1}$ & $u-g, g-r, r-i, i-z$ \\
\hline 3 & $5 \pm{ }_{2}^{77}$ & $0.4 \pm 0.1$ & $\mathrm{NUV}-g, u-g, g-r, r-i$ \\
\hline 4 & $87 \pm{ }_{83}^{48}$ & $0.02 \pm \pm_{0.02}^{0.48}$ & $\mathrm{NUV}-g, g-r, r-i$ \\
\hline \multicolumn{4}{|c|}{ NGC 2856 disk clumps } \\
\hline $1^{\mathrm{b}}$ & $192 \pm \underset{186}{2108}$ & $0.4 \pm \pm_{0.4}^{0.38}$ & $u-g, g-r, r-i$ \\
\hline 2 & $9 \pm_{3}^{79}$ & $0.56 \pm{ }_{0.08}^{0.2}$ & $u-g, g-r, r-i, i-z$ \\
\hline $3^{\mathrm{c}}$ & $1600 \pm \begin{array}{l}8500 \\
1596\end{array}$ & $0.18 \pm \underset{0.1}{1.22}$ & $u-g, g-r, r-i, i-z$ \\
\hline 4 & $102 \pm{ }_{96}^{1798}$ & $0.56 \pm \begin{array}{l}0.38 \\
0.56\end{array}$ & $u-g, g-r, r-i, i-z$ \\
\hline \multicolumn{4}{|c|}{ NGC 2854 disk clumps } \\
\hline 1 & $5 \pm{ }_{2}^{15}$ & $0.3 \pm{ }_{0.3}^{0.2}$ & $\mathrm{NUV}-g, u-g, g-r, r-i, i-z$ \\
\hline 2 & $9 \pm{ }_{5}^{6791}$ & $0.6 \pm 0.4$ & $u-g, g-r, r-i, i-z$ \\
\hline 3 & $3 \pm 3$ & $0.6 \pm 0.5$ & $u-g, g-r, r-i, i-z$ \\
\hline 4 & $6 \pm \pm_{1}^{2}$ & $0.88 \pm{ }_{0.22}^{0.08}$ & $u-g, g-r, r-i, i-z$ \\
\hline 5 & $7 \pm{ }_{3}^{2293}$ & $0.6 \pm 0.6$ & $u-g, g-r, r-i, i-z$ \\
\hline 6 & $10 \pm{ }_{6}^{10090}$ & $0.4 \pm{ }_{0.4}^{0.6}$ & $u-g, g-r, r-i, i-z$ \\
\hline $7^{\mathrm{c}}$ & $7000 \pm \begin{array}{l}3100 \\
5300\end{array}$ & $0.48 \pm{ }_{0.16}^{0.12}$ & $u-g, g-r, r-i, i-z$ \\
\hline 8 & $1400 \pm{ }_{300}^{700}$ & $0.1 \pm_{0.1}^{0.2}$ & $u-g, g-r, r-i$ \\
\hline
\end{tabular}

Notes.

a All clump ages obtained with instantaneous burst models. The oldest models run were $10 \mathrm{Gyr}$ old.

b "Bright spot" in northwestern edge of disk. Assuming 0.2 solar metallicty, the upper limit on the NUV flux does not constrain this age further.

c Nucleus.

Spitzer grant 1263924, NSF grant AST-0097616, NASA LTSA grant NAG5-13079, and NASA GALEX grant GALEXGI040000-0026. V. C. acknowledges partial support from the EU ToK grant 39965. We thank Jayaram Chengalur for providing us with an electronic copy of the $\mathrm{H}$ i data. We also acknowledge Amanda Moffett and Chris Carver for help with system administration. This research has made use of the NASA/IPAC Extragalactic Database (NED) which is operated by the Jet Propulsion Laboratory, California Institute of Technology, under contract with the National Aeronautics and Space Administration.

\section{APPENDIX}

In this Appendix, we provide additional color-color plots for the star-forming clumps in Arp 285, as well as additional timesteps for the numerical simulation of the interaction and alternative results for the population synthesis modeling of the clumps. In Figure A1, the $r-i$ color of the clumps is plotted against $g-r$, while in Figure A2, $i-z$ vs. $r-i$ is presented. Figure A3 shows FUV-NUV plotted against $g-r$, and Figure A4 gives [5.8]-[8.0] vs. [4.5]-[5.8]. Figure A5 shows two later timesteps in the numerical simulation of the interaction. Table A1 gives age and extinction estimates for the clumps, assuming 0.2 solar metallicity population synthesis models.

\section{REFERENCES}

Abazajian, K., et al. 2003, AJ, 126, 2081

Abraham, R. G., \& van den Bergh, S. 2001, Science, 293, 1273

Arp, H. 1966, in Atlas of Peculiar Galaxies (Pasadena, CA: Caltech) 
Barnes, J. E., \& Hernquist, L. 1992, Nature, 360, 715

Bastian, N., Gieles, M., Efremov, Y. N., \& Lamers, H. J. G. L. M. 2005, A\&A, 443, 79

Bohlin, R. C., Savage, B. D., \& Drake, J. F. 1978, ApJ, 224, 132

Braine, J., Duc, P.-A., Lisenfeld, U., Charmandaris, V., Vallejo, O., Leon, S., \& Brinks, E. 2001, A\&A, 378, 51

Bushouse, H. A., Lamb, S. A., \& Werner, M. W. 1988, ApJ, 335, 74

Calzetti, D., Kinney, A. L., \& Storchi-Bergmann, T. 1994, ApJ, 429, 582

Calzetti, D., et al. 2005, ApJ, 633, 871

Cerviño, M., Valls-Gabaud, D., Luridiana, V., \& Mas-Hess, J. M. 2002, A\&A, 381, 51

Chen, C., \& Wu, H. 2007, AJ, 133, 1710

Chengalur, J. N., Salpeter, E. E., \& Terzian, Y. 1994, AJ, 107, 1984

Chengalur, J. N., Salpeter, E. E., \& Terzian, Y. 1995, AJ, 110, 167

Cutri, R. M., et al. 2006, Explanatory Supplement to the 2MASS All Sky Release and Extended Mission Products, http://www.ipac.caltech.edu/2mass/

Draine, B. T., \& Li, A. 2007, ApJ, 657, 810

Duc, P.-A., Brinks, E., Wink, J. E., \& Mirabel, I. F. 1997, A\&A, 326,537

Duc, P.-A., \& Mirabel, I. F. 1994, A\&A, 289, 83

Elmegreen, B. G., \& Efremov, Y. N. 1996, ApJ, 466, 802

Elmegreen, D. M., Elmegreen, B. G., Kaufman, M., Sheth, K., Struck, C., Thomasson, M., \& Brinks, E. 2006, ApJ, 642, 158

Elmegreen, B. G., Kaufman, M., \& Thomasson, M. 1993, ApJ, 412, 90

Fazio, G. G., et al. 2004, ApJS, 154, 10

Feldmeier, J. J., et al. 2002, ApJ, 575, 779

Flagey, N., Boulanger, F., Vrestraete, L., Miville Deschênes, M. A., Noriega Crespo, A., \& Reach, W. T. 2006, A\&A, 453, 969

Hancock, M., Smith, B. J., Struck, C., Giroux, M. L., Appleton, P. N., Charmandaris, V., \& Reach, W. T. 2007, AJ, 133, 676

Higdon, S. J. U., Higdon, J. L., \& Marshall, J. 2006, ApJ, 640, 768

Holtzman, J. A., et al. 1992, AJ, 103, 691

Holtzman, J. A., et al. 1996, AJ, 112, 416

Hunter, D. A., \& Gallagher, J. S. III 1985, ApJS, 58, 533

Jester, S., et al. 2005, AJ, 130, 873

Karachentsev, I. D., Karachentseva, V. E., Huchtmeier, W. K., \& Makarov, D. I. 2004, AJ, 127, 2031

Kennicutt, R. C. Jr., et al. 1987, AJ, 93, 1011

Kroupa, P. 2002, Science, 295, 85

Larsen, S. S. 2004, A\&A, 416, 537

Leitherer, C., et al. 1999, ApJS, 123, 3

Li, H., Wu, H., Cao, C., \& Zhu, Y. 2007, AJ, 134, 1315
MacArthur, L. A., Courteau, S., Bell, E., \& Holtzman, J. A. 2004, ApJS, 152,175

Martin, D. C., et al. 2005, ApJ, 619, L1

McLaughlin, D., \& van der Marel, R. P. 2005, ApJ, 161, 304

Morris, S. L., \& van den Bergh, S. 1994, ApJ, 427, 696

O’Connell, R. W., Gallagher, J. S., \& Hunter, D. A. 1994, ApJ, 433, 65

Pasquali, A. de Grijs R., \& Gallagher, J. S. 2003, MNRAS, 345, 161

Patterson, R. J., \& Thuan, T. X. 1996, ApJS, 107, 103

Press, W. H., Teukolsky, S. A., Vetterling, W. T., \& Flannery, B. P. 1992 Numerical Recipes in Fortran (2nd ed.; Cambridge: Cambridge Univ. Press), 692

Reshetnikov, V., Bournaud, F., Combe, F., Faúndez-Abans, M., \& de OliveiraAbans, M. 2006, A\&A, 446, 447

Rieke, G. H., et al. 2004, ApJS, 154, 25

Roche, N. 2008, arXiv astro-ph/0605015

Sanders, D. B., Soifer, B. T., Elias, J. H., Madore, B. F., Matthews, K., Neugebauer, G., \& Scoville, N. Z. 1988, ApJ, 325, 74

Schweizer, F., Miller, B. W., Whitmore, B. C., \& Fall, S. M. 1996, AJ, 112, 1839

Smith, B. J., Kleinmann, S. G., Huchra, J. P., \& Low, F. 1987, ApJ, 318, 161

Smith, B. J., Struck, C., Appleton, P. N., Charmandaris, V., Reach, W., \& Eitter, J. J. 2005b, AJ, 130, 2117

Smith, B. J., Struck, C., Hancock, M., Appleton, P. N., Charmandaris, V., \& Reach, W. 2007, AJ, 133, 791

Smith, B. J., Struck, C., \& Nowak, M. A. 2005a, AJ, 129, 1350

Smith, B. J., Struck, C., \& Pogge, R. W. 1997, ApJ, 483, 754

Soifer, B. T., Sanders, D. B., Madore, B. F., Neugebauer, G., Danielson, G. E., Elias, J. H., Lonsdale, C. J., \& Rice, W. L. 1987, ApJ, 320, 238

Struck, C. 1997, ApJS, 113, 269

Struck, C., Kaufman, M., Brinks, E., Thomasson, M., Elmegreen, B. G., \& Elmegreen, D. M. 2005, MNRAS, 364, 69

Struck, C., \& Smith, B. J. 2003, ApJ, 589, 157

Toomre, A., \& Toomre, J. 1972, ApJ, 178, 623

Vázquez, G. A., \& Leitherer, C. 2005, ApJ, 621, 695

Wallin, J. F., \& Struck-Marcell, C. 1988, AJ, 96, 1850

Watson, A. M., et al. 1996, AJ, 112, 534

Weilbacher, P. M., Duc, P. A., \& Fritze-v. Alvensleben, U. 2003, A\&A, 397, 545

Werner, M. W., et al. 2004, ApJS, 154, 1

Whitmore, B. C., \& Schweizer, F. 1995, AJ, 109, 960

Whitmore, B. C., Schweizer, F., Leitherer, C., Borne, K., \& Robert, C. 1993, AJ, 106,1354

Whitney, B. A., et al. 2004, ApJS, 154, 315

Zhang, Q., Fall, S. M., \& Whitmore, B. C. 2001, ApJ, 561, 727 